\title{
Kompetenzerwerb und Meinungsbildung in Bürgerkonferenzen
}

\section{Kurzfassung}

Die Wirkung von Bürgerbeteiligungsverfahren kann für verschiedene Bereiche untersucht werden: für die Öffentlichkeit, für die Politik, für die Wirtschaft oder für die Wissenschaft. Politische Partizipationsverfahren können aber ebenso gut Einflüsse auf die teilnehmenden Bürger ausüben. Nach dem bisherigen Forschungsstand können zwei Dimensionen ausgemacht werden: Kompetenzerwerb und Meinungsbildung bzw. Meinungsänderung. Mittels empirischer Daten einer standardisierten Befragung im Rahmen des „Bürgerdialogs Zukunftsthemen“ des Bundesministeriums für Bildung und Forschung werden unterschiedliche Effekte auf die teilnehmenden Bürger ermittelt. Darüber hinaus werden Gruppenunterschiede nach den Variablen Geschlecht, Alter und Bildung untersucht.

Die Ergebnisse bestätigen Wirkungseffekte auf beiden Dimensionen: Durch ihre Teilnahme erlangen die Bürger neues Wissen, ihr Interesse am Thema steigt und ihre Meinungen zum Thema ändern sich. Insgesamt zeigt sich, dass es bei Beteiligungsverfahren um mehr geht als um politische Mitbestimmung. 


\section{Inhalt}

1. Einleitung 40

2. Der Bürgerdialog „Energietechnologien für die Zukunft“

3. Evaluationskonzept 42

4. Zur Wirkung von Partizipationsverfahren: Der Forschungsstand 43

a) Wirkung als Evaluationskriterium von Partizipationsverfahren 44

b) Dimensionen der Wirkungsanalyse 45

c) Unterschiede in den Wirkungen nach spezifischen Gruppen 47

5. Wirkung der Bürgerkonferenzen des Bürgerdialogs „Energietechnologien für die Zukunft" auf die Bürger $\quad 48$

a) Dimension: Kompetenzerwerb 49

(1) Erwerb von Fakten- und Sachwissen 49

(2) Zunahme an Interesse 53

(3) Informeller Lernerfolg $\quad 54$

(4) Fazit Kompetenzerwerb 58

b) Dimension: Meinungsbildung bzw. -änderung 58

(1) Meinung über Wissenschaftler und Politiker 59

(2) Bürgerbeteiligung in Deutschland 59

(3) Standpunkt zum Thema 65

(4) Fazit Meinungsbildung und -änderung 67

6. Diskussion der Ergebnisse $\quad 67$

7. Fazit 71

\section{Einleitung}

Das Bundesministerium für Bildung und Forschung (BMBF) hat im Jahr 2011 den „Bürgerdialog Zukunftsthemen“ ins Leben gerufen (http://www.buergerdialogbmbf.de/). Der Bürgerdialog bietet Bürgern ${ }^{1}$ die Möglichkeit,

- sich über Zukunftsthemen zu informieren

- sich im offenen Austausch mit Experten eine fundierte Meinung zu bilden

- mit Vertretern aus Politik, Wirtschaft, Wissenschaft und Gesellschaft zu diskutieren

- Meinungsbilder und Handlungsempfehlungen für Politik, Wirtschaft, Wissenschaft und Gesellschaft zu formulieren.

1 Zugunsten der einfacheren Lesbarkeit wird im Folgenden ausschließlich die männliche Form verwendet. Gemeint sind jedoch beide Geschlechter. 
In der Projektlaufzeit fanden drei Bürgerdialoge zu verschiedenen Themen statt. 2011 wurden die beiden Bürgerdialoge „Energietechnologien für die Zukunft“ und „Hightech-Medizin“ durchgeführt. Der dritte Bürgerdialog 2012/2013 dreht sich um die Frage, wie Deutschland in Zeiten des demografischen Wandels ideenreich und innovativ bleibt. Das zentrale Element jedes Bürgerdialogs ist eine Reihe regionaler Bürgerkonferenzen, bei denen bis zu 100 Bürger über das jeweilige Thema diskutieren können.

Im Folgenden wird anhand des ersten Bürgerdialogs über Energietechnologien die Frage diskutiert: Welche Wirkung hat die Teilnahme an einer Bürgerkonferenz auf die Bürger? Doch bevor diese Frage genauer beleuchtet wird, soll das Konzept des Bürgerdialogs und das Evaluationsdesign, auf dem der Artikel empirisch fußt, vorgestellt werden.

\section{Der Bürgerdialog „Energietechnologien für die Zukunft““}

Mit dem Bürgerdialog Energietechnologien soll Bürgern die Gelegenheit gegeben werden, über den Beitrag zukunftsrelevanter Technologien mit Experten zu diskutieren, ihre Fragen und Bedenken zu formulieren und sich somit aktiv an der politischen Diskussion zu beteiligen. Methodisch baut der Dialog auf vier Bausteinen auf:

1. Regionale Bürgerkonferenzen: Eintägige regionale Bürgerkonferenzen sind das Kernelement des Bürgerdialogs. Jeweils bis zu 100 nach repräsentativen Gesichtspunkten ausgewählte Bürger kommen zusammen mit dem Ziel, Handlungsansätze für den politischen und gesellschaftlichen Umgang mit dem jeweiligen Zukunftsthema zu formulieren. Die erste Kontaktaufnahme mit den Bürgern erfolgt nach einer Zufallsauswahl. Danach werden die Teilnehmer im Sinne einer ausgewogenen Zusammenstellung nach Alter, Geschlecht und Bildungsstand ausgewählt. Bereits im Vorfeld der regionalen Bürgerkonferenzen erhalten die Teilnehmer Informationsmaterial über das Thema.

Eingeladen werden neben den Bürgern zwischen drei und fünf Fachexperten, die je nach Bedarf mit den Bürgern diskutieren und bei Fragen zum Thema aufklären. Das zentrale Element der Bürgerkonferenzen sind die Tischdiskussionen. An runden Tischen mit je maximal zehn Personen entwickeln die Bürger unter der Leitung eines Moderators Handlungsoptionen für die Politik. Im Ergebnis endet jede Bürgerkonferenz mit einem Dokument, das zu allen Schwerpunktthemen der Veranstaltung Stellung nimmt und Zukunftskorridore für das Themenfeld aufzeigt. 
Beim Bürgerdialog Energietechnologien wurden acht Bürgerkonferenzen in verschiedenen Regionen Deutschlands durchgeführt (z. B. Berlin, Frankfurt, Stuttgart, Hamburg).

2. Online-Dialog: Während des gesamten Dialogs haben alle interessierten Bürger die Möglichkeit, sich über die Webseite des Bürgerdialogs zum Dialogthema zu informieren und online über verschiedene Thesen zum Thema mitzudiskutieren.

3. Bürgergipfel: Auf dem abschließenden Bürgergipfel erarbeiten Bürger auf Basis der Ergebnisse aus den Bürgerkonferenzen und dem Online-Dialog den Bürgerreport. Dieser enthält konkrete Handlungsempfehlungen zum Thema Energie und wird an einen Vertreter des BMBF übergeben.

4. Beraterkreis: Der Beraterkreis ist mit Experten aus Wirtschaft, Wissenschaft und Zivilgesellschaft sowie ausgewählten Bürgern besetzt. Er begleitet, unterstützt und berät den gesamten Bürgerdialog.

\section{Evaluationskonzept}

Der gesamte Prozess des Bürgerdialogs wird von einer an der Konzeption und Durchführung des Bürgerdialogs nicht beteiligten Institution evaluiert. Die verwendeten Methoden zur Evaluation der verschiedenen Bausteine des Bürgerdialogs sind in der folgenden Übersicht aufgeführt.

Tabelle 1: Evaluationsdesign

\begin{tabular}{|l|l|}
\hline Baustein & Vorgehen bei der Evaluation \\
\hline $\begin{array}{l}\text { Bürgerkonferenzen und } \\
\text { Bürgergipfel }\end{array}$ & $\begin{array}{l}\text { Standardisierte Befragung der Teilnehmer in drei Wellen } \\
\text { Teilnehmende Beobachtungen }\end{array}$ \\
\hline Online-Dialog & $\begin{array}{l}\text { Standardisierte Befragung der registrierten Teilnehmer } \\
\text { Dokumentenanalyse }\end{array}$ \\
\hline Beraterkreis & Leitfadengestützte Interviews \\
\hline
\end{tabular}

Quelle: Eigene Darstellung.

Das primäre Instrument der Evaluation der Bürgerkonferenzen und empirische Grundlage dieses Artikels ist die standardisierte Befragung in drei Wellen. Dabei werden alle teilnehmenden Bürger der Bürgerkonferenzen gebeten, Fragebögen auszufüllen. Die erste Befragung findet unmittelbar vor der Veranstaltung, die zweite unmittelbar danach und die dritte ca. zwei Monate nach der Veranstaltung statt. Die Evaluationskriterien entsprechen weitgehend den anerkannten und üblichen Kriterien: Offenheit, Transparenz, Fairness, Effizienz und Effektivität (Kersting 2008). Die Themen der ersten Befragung umfassen die Motivation der Teilnahme, Einschätzungen zur Bürgerbeteiligung in Deutschland, Fragen zur Energie- 
wende, Erwartungen an den Bürgerdialog, Bewertung des Vorbereitungsmaterials sowie soziodemografische Angaben. In der zweiten Welle geht es vor allem um die Zufriedenheit mit der Bürgerkonferenz, die Bewertung der Gesprächskultur und der Ergebnisse sowie um die Wirkung der Veranstaltung. Der Fragebogen der dritten Welle greift Themenkomplexe der ersten und zweiten Welle wieder auf, vor allem die Fragen zur Zufriedenheit und zur Wirkung der Veranstaltung. Die Befragung in drei Wellen soll die zeitliche Entwicklung von Meinungen dokumentieren. Insgesamt wurden von den 791 Teilnehmern bei den Bürgerkonferenzen 688 Bürger in Welle 1, 630 Bürger in Welle 2 und 323 Bürger in Welle 3 befragt.

Bevor die Ergebnisse der Evaluation unter der hier vorliegenden Fragestellung präsentiert werden, soll ein Überblick über den aktuellen Forschungstand zum Thema Wirkung von Beteiligungsverfahren gegeben werden.

\section{Zur Wirkung von Partizipationsverfahren: Der Forschungsstand}

Die Wirkung von Partizipationsverfahren kann für verschiedene Akteure untersucht werden: beispielsweise für die Öffentlichkeit, für die Politik, für die Wirtschaft oder für die Wissenschaft. Im Folgenden sollen nur die Studien herausgearbeitet werden, die sich mit der Wirkung auf die teilnehmenden Bürger bei Partizipationsverfahren beschäftigt haben. Politische Partizipationsverfahren werden hier im Sinne der Politik- und Governanceperspektive definiert als Formen der öffentlichen Entscheidungsfindung, bei der nichtstaatliche Akteure, die nicht regulär an politischen Entscheidungsprozessen teilnehmen und die als Repräsentanten von Betroffenen agieren, einen substanziellen Einfluss auf eine kollektiv-verbindliche Entscheidung nehmen (Newig 2011: 67).

Die Bürgerkonferenzen im Bürgerdialog „Energietechnologien für die Zukunft“ können zu den Partizipationsverfahren gezählt werden. Derartige Verfahren gewinnen in den letzten Jahren zunehmend an Bedeutung (Kersting 2008). Allerdings wird der Begriff der Bürgerkonferenz in der Literatur nicht einheitlich verwendet und beispielsweise mit Konsensus- oder Abstimmungskonferenzen gleichgesetzt (Burow/Kühnemuth 2004; Zimmer 2002). Gemeinsam ist diesen Verfahren, dass sie auf einer repräsentativen Stichprobe beruhen und unter der Leitung eines Moderators durchgeführt werden. ${ }^{2}$ Theoretisch können sie auf das Demokratiemodell der Deliberativen Demokratie zurückgeführt werden (ausführlicher Habermas 1992; Lösch 2005). In dem vorliegenden Artikel werden Bürgerkonferenzen im engen Verständnis des Bürgerdialogs „Energietechnologien für die Zukunft“ als

2 Auch Deliberative Polls, Planungszellen oder Citizen Jurys beruhen auf einem ähnlichen Design. Kersting (2008) nennt diese Verfahren ,Jury“. 
eintägige Veranstaltungen mit einem repräsentativ zusammengesetzten Teilnehmerkreis verstanden. Bei Konsensus- oder Abstimmungskonferenzen treffen sich die Bürger dagegen häufiger. Zu vermuten ist deshalb, dass hier andere Wirkungseffekte auftreten (Hebestreit 2013: 188), die im Rahmen dieses Artikels nicht weiter untersucht werden können.

\section{a) Wirkung als Evaluationskriterium von Partizipationsverfahren}

Die empirische Analyse der Wirkung von Partizipationsverfahren auf die Bürger findet in der Regel im Rahmen von Evaluationen statt. Allerdings gibt es bis heute keinen allgemein anerkannten Kriterienkatalog für Evaluationen von Beteiligungsprojekten. ${ }^{3}$ Im Allgemeinen wird zwischen Prozess- und Ergebniskriterien unterschieden (ausführlicher Kersting 2008). Bei Prozessevaluationen geht es häufig um die Aspekte Inklusion, Gerechtigkeit, Effizienz, Transparenz und Prozessqualität (Kersting 2008; Abelson u. a. 2003; Rowe/Frewer 2000). Die Ergebnisevaluation beschäftigt sich vor allem mit der Analyse der Wirkung bzw. mit den Lernerfolgen auf die beteiligten Akteure (ausführlicher Beierle/Cayford 2002).

Theoretisch werden diese Effekte mit verschiedenen Lerntheorien untermauert. Zentrale Begriffe sind hier das kooperative Lernen (Green/Green 2006), soziales Lernen (Reed u. a. 2010) und gesellschaftliches Lernen (Stoltenberg 2007). Beim gesellschaftlichen Lernen geht es um jene Aspekte, in denen im weitesten Sinn die sozialen und strukturellen Aspekte von Gesellschaft in den Mittelpunkt rücken. Für den vorliegenden Artikel ist das kooperative Lernen zentraler. Ziel ist es, durch das gemeinsame Bearbeiten einer Aufgabe einen Konsens bzw. ein gemeinsames Statement zu finden. Voraussetzung dafür ist, dass die Bürger gemeinsam an der Aufgabe arbeiten, persönlich miteinander interagieren, idealerweise in Kleingruppen, und jeder Teilnehmer sich einbringt. Dadurch lernen die Teilnehmer Fragen zu stellen, zuzuhören, die eigene Meinung zu formulieren und gemeinsam mit anderen Personen Lösungen zu entwickeln. Die Meinungen anderer müssen dabei respektiert werden. Insofern kann soziales und kognitives Lernen unter kooperatives Lernen subsumiert werden. Die Bedingungen kooperativen Lernens sind bei politischen Partizipationsformaten wie Konsensus- oder Bürgerkonferenzen in den Konzepten angelegt. Doch welche konkreten Wirkungseffekte bzw. Lernerfolge auf die teilnehmenden Bürger werden in der bisherigen Literatur für solche Verfahren untersucht?

3 Ein Versuch allgemeiner Kriterien z. B. in Rowe/Frewer 2000, Rowe/Marsh/Frewer 2004; Holtkamp/Bogumil/Kißler 2006. Siehe auch Geißel 2008; Kersting 2008; Kubicek/Lippa/Koop 2011. 


\section{b) Dimensionen der Wirkungsanalyse}

Grundsätzlich herrscht Einigkeit darüber, dass die politische Einbindung von Bürgern im Rahmen von Partizipationsverfahren Lernprozesse in Gang setzen kann. ${ }^{4}$ Die Literatur zeigt eine Vielfalt möglicher Effekte auf. Die zentralen Schlagworte sind Wissen (Wissenschaft im Dialog 2011), Interesse (ebd.), politisches Engagement (Barber 1984), Meinungsänderung (Talpin/Wojcik 2010), Verständnis für die Meinung anderer (Gutmann/Thompson 1996) und die Fähigkeit, die eigene Meinung zu artikulieren (Chambers 1996). Diese verschiedenen Lerneffekte, hier im Weiteren etwas allgemeiner als Wirkungseffekte auf die teilnehmenden Bürger bezeichnet, können in zwei verschiedene Dimensionen geclustert werden:

1. Kompetenzerwerb; ${ }^{5}$

2. Meinungsbildung bzw. -änderung. ${ }^{6}$

Die erste Dimension der Wirkungsanalyse, der Kompetenzerwerb, behandelt vor allem die Frage, was die Teilnehmer Neues erfahren und lernen. Dabei stehen drei Kriterien im Mittelpunkt:

- Erwerb von Fakten- und Sachwissen: Hier ist die Frage zentral, ob die teilnehmenden Bürger neues Wissen zum Thema erlangt haben. Gemessen wird dieses Kriterium in der Regel über die Items subjektive Wissenseinschätzung und Informiertheit. Der Erwerb von Fakten- oder Sachwissen über das inhaltliche Thema gilt dabei als Voraussetzung, um zu einem fundierten Urteil in Bezug auf das Thema des Beteiligungsverfahrens zu gelangen. So können Bürger ,übergeordnetes Wissen (Metawissen) erlangen, zum Beispiel über die richtige Deutung von Fakten und wissenschaftlichen Ergebnissen" (Wissenschaft im Dialog 2011: 100). Insbesondere Veranstaltungen, die über einen längeren Zeitraum laufen (z. B. Konsensuskonferenzen) und Experten einbinden, erzielen nach aktuellen Studien einen Zuwachs an Wissen und Informiertheit bei den Teilnehmern. ${ }^{7}$

- Zunahme an Interesse: Der Kompetenzerwerb wird auch über die Zunahme des themenspezifischen Interesses und der intrinsischen Motivation, sich weiter mit dem Thema zu beschäftigen, gemessen. Die bisherigen Studien zeigen, dass bei

4 Siehe Wissenschaft im Dialog 2011; Delli Carpini/Cook/Jacobs 2004; Gutmann/Thompson 1996; Mendelberg 2002; Chambers 1996; Talpin/Wojcik 2010; Zimmer 2002; Luskin/Fishkin/Jowell 2002.

5 Vergleiche Andersen/Hansen 2007; Zimmer 2002; Marxer 2004; Sellke/Renn/Cornelisse 2007; Gohl/Wüst 2008; Wissenschaft im Dialog 2011; Talpin/Wojcik 2010; Mayer/de Vries/Geurts 1995.

6 Etwa Talpin/Wojcik 2010; Mayer/de Vries/Geurts 1995; Zimmer 2002; Wissenschaft im Dialog 2011.

7 Siehe hierzu etwa Wissenschaft im Dialog 2011; Zimmer 2002; Talpin/Wojcik 2010; Mayer/de Vries/Geurts 1995. 
vielen Teilnehmern nach der Veranstaltung das thematische Interesse zunimmt (Wissenschaft im Dialog 2011; Stollen 2011). Darüber hinaus zeigen Befragungen einige Monate nach der Veranstaltung, dass die Teilnehmer in Eigeninitiative weitere Informationen recherchieren und angeben, mit anderen nach der Veranstaltung häufiger über das Diskussionsthema zu kommunizieren (Wissenschaft im Dialog 2011).

- Informeller Lernerfolg: Neben dem Erwerb von inhaltsspezifischem Interesse und Fachwissen wird auch der Aspekt des informellen Lernens aufgegriffen. Dieser Begriff stammt aus Analysen der Effekte des ehrenamtlichen Engagements (Gensicke/Picot/Geiss 2006). Im Wesentlichen wird darunter die Verbesserung der sozialen und organisatorischen Kompetenzen verstanden. Dazu gehören beispielsweise rhetorische Fertigkeiten oder die Fähigkeit, in Konflikten zu vermitteln. Im Hinblick auf die Wirkung von Partizipationsprojekten können drei verschiedene Arten des informellen Lernens unterschieden werden: die Aneignung sozialer, demokratischer und wissenschaftlicher Kompetenzen. Bei den sozialen Kompetenzen geht es vor allem um Effekte auf die Fähigkeit, eigene Präferenzen zu kommunizieren, Toleranz für andere Meinungen zu entwickeln und gemeinsam mit anderen zu einem Urteil zu kommen (Gutmann/Thompson 1996; Chambers 1996; Schophaus/Dienel 2002). Demokratische Kompetenz meint Lernerfolge, die sich auf die Bereitschaft der Beteiligten beziehen, sich an politischen Diskussionen aktiv zu beteiligen (Barber 1984; Wissenschaft im Dia$\log$ 2011). Effekte auf die wissenschaftliche Kompetenz werden zum Beispiel über die grundsätzliche Aufgeschlossenheit gegenüber wissenschaftlichen Fragestellungen und Methoden untersucht (Wissenschaft im Dialog 2011).

Bei der zweiten Dimension der Wirkungsanalyse geht es um die Meinungsbildung bzw. Meinungsänderung. Diese kann sich auf das Thema, aber auch auf die Meinung gegenüber dem politischen System oder der Wissenschaft beziehen. Vereinzelt wird auch die Möglichkeit der Akzeptanzschaffung von Maßnahmen diskutiert (Kubicek/Lippa/Koop 2011).

- Meinung zum Thema: Insgesamt weisen die Ergebnisse hinsichtlich der Meinung zum Thema der Veranstaltung in unterschiedliche Richtungen. Während eine Gruppe von Autoren davon spricht, dass sich verfestigte Meinungen nur sehr schwer ändern lassen und daher eher keine Meinungsänderung zustande kommt (Talpin/Wojcik 2010), beschreibt eine andere Gruppe von Autoren, dass sich Meinungen durch Bürgerbeteiligungsverfahren in Richtung einer kritischeren Haltung verändern (Mayer u. a. 1995). Zimmer (2002) und Wissenschaft im Dialog (2011) berichten folgende Ergebnisse: Haben sich Personen bereits eine Meinung bezüglich eines bestimmten Themas gebildet, so wird sich diese durch 
die Teilnahme an Beteiligungsverfahren in der Regel verfestigen. Die Informations- und Wissensvermittlung führt jedoch bei einer Vielzahl von Personen, die sich noch keine Meinung gebildet haben, zu einem differenzierten Meinungsbild.

- Meinung zum politischen System: Bezüglich der Meinung gegenüber dem politischen System ist Politikverdrossenheit das Schlagwort, denn nur die Minderheit der Deutschen hält ihren eigenen Einfluss auf politische Entscheidungen für stark (Gabriel/Holtmann 2005). Es gibt aber Indizien dafür, dass Bürger unmittelbar nach der Teilnahme an partizipativen Verfahren eher glauben, dass sich Bürger sinnvoll in politische Entscheidungen einbringen können (Goldschmidt/Scheel/ Renn 2012). Ob diese Meinungsänderung langfristig Bestand hat, kann damit aber nicht gesagt werden.

Insgesamt kann festgehalten werden, dass die Wirkung von Bürgerbeteiligungsverfahren auf die Bürger in der Regel über den Kompetenzerwerb, der sich neben dem Faktenwissen und der Interessenszunahme auf soziale, wissenschaftliche und demokratische Kompetenzen bezieht, sowie über die Meinungsbildung bzw. -änderung untersucht wird. Im Ergebnis schlussfolgern viele Studien, dass Partizipationsverfahren informierte und kompetente Bürger hervorbringen können.

Mittlerweile werden die Lernerfolge solcher Beteiligungsverfahren im Vergleich zwischen Face to face-Verfahren und Online-Verfahren untersucht (Talpin/Wojcik 2010; Min 2007). Dabei zeigen Online-Verfahren ähnliche positive Effekte, wie zum Beispiel Lernanstöße beim Wissen, gesteigertes politisches Vertrauen und ein gesteigerter Wille, sich aktiv politisch zu beteiligen. Vor allem durch das Surfen auf der Website lernen die Bürger Neues - weniger durch das Lesen eingestellter Dokumente oder der inhaltlichen Diskussion (Talpin/Wojcik 2010). Die Ergebnisse bezüglich der Meinungsänderungen durch Online-Verfahren sind unterschiedlich. Luskin u. a. (2004) zeigen Effekte auf die Meinungsbildung, bei Min (2007) dagegen gibt nur ein kleiner Teil der Befragten derartige Effekte an. Insgesamt sind die Lernerfolge bei Online-Verfahren etwas geringer als bei Face to face-Verfahren. Der größte Effekt zeigt sich aber, wenn Bürger über Online- und Face to face-Verfahren eingebunden werden.

\section{c) Unterschiede in den Wirkungen nach spezifischen Gruppen}

Die Wirkungsanalyse auf die beteiligten Bürger erfolgt in der Regel auf der allgemeinen Ebene. Spezielle Gruppenvergleiche finden nicht statt. Diese Forschungslücke möchten wir mit dem vorliegenden Artikel explorativ beleuchten. Anhand von drei in der sozialwissenschaftlichen Forschung typischen Kontroll- bzw. Erklärungsvariablen, nämlich Geschlecht, Alter und Bildung, möchten wir Gruppen- 
vergleiche durchführen. ${ }^{8}$ Bei diesen Variablen sind gruppenspezifische Wirkungseffekte zu vermuten.

- Geschlecht: Aus der Bildungsforschung ist bekannt, dass Männer und Frauen unterschiedlich kommunizieren und lernen. ${ }^{9}$ Frauen zeigen ein größeres kooperatives Verhalten und können Themenkomplexe eher in der Gruppe bearbeiten. In gemischtgeschlechtlichen Gruppen setzen sich allerdings die Männer durch. Sie reden häufiger, länger und unterbrechen andere eher. Da bei partizipativen Verfahren typischerweise keine monoedukativen Gruppen gebildet werden, prallen diese genderspezifischen Lernkulturen aufeinander. Diese Vermutung soll im Folgenden mit Blick auf die unterschiedlichen Auswirkungen auf den Lerneffekt untersucht werden.

- Alter und Bildung: Ergebnisse aus Studien aus dem ehrenamtlichen Engagement lassen vermuten, dass vor allem jüngere Personen über Lernerfolge, speziell über informelle Aspekte, nach der Teilnahme an Partizipationsverfahren berichten (Gensicke/Picot/Geiss 2006). Lernerfolge dieser Gruppe werden vor allem auf mangelnde Erfahrungen zurückgeführt. Möglicherweise hängen aus diesem Grund auch Alter und Bildung hier zusammen und die Lerneffekte sind bei jüngeren Personen mit niedriger Bildung am stärksten ausgeprägt.

Diese Vermutungen sollen anhand der Bürgerkonferenzen zum Thema „Energietechnologien für die Zukunft" explorativ diskutiert werden.

\section{Wirkung der Bürgerkonferenzen des Bürgerdialogs „Energietechnologien für die Zukunft" auf die Bürger}

Im Folgenden soll die Frage untersucht werden, welche Wirkung bei den teilnehmenden Bürgern an den Bürgerkonferenzen des Bürgerdialogs „Energietechnologien für die Zukunft“ gemessen werden kann. Konkret geht es um folgende Forschungsfragen:

- Welche Effekte lassen sich auf die teilnehmenden Bürger der Bürgerkonferenzen feststellen?

- Unterscheiden sich die Effekte direkt nach der Veranstaltung und zwei Monate später?

8 Diese Variablen werden bei Regressionsmodellen häufig als Proxy-Variablen eingesetzt. Dahinter verbirgt sich die Annahme, dass diese Variablen für mehr stehen als den objektiven Bildungsabschluss, das biologische Geschlecht und Alter. Somit können latente Konstrukte indirekt abgebildet werden (Urban/Mayerl 2008: $220 \mathrm{f}$.).

9 Siehe beispielsweise Schmidt 1988; Werner 1983; Niehoff 1993; Derichs-Kunstmann 2001. 
- Gibt es bezüglich der Variablen Geschlecht, Alter und Bildung Auffälligkeiten bei der Analyse der Effekte?

Alle folgenden empirischen Analysen beziehen sich auf diejenigen Teilnehmer der Bürgerkonferenzen, die an allen drei Erhebungswellen teilgenommen haben und deren Ergebnisse über einen persönlichen Code zweifelsfrei einander zugeordnet werden konnten. Damit ergibt sich eine maximale Fallzahl von 179. Statistisch konzentrieren wir uns dabei auf Mittelwertvergleiche, die Angabe der Signifikanzniveaus und auf Regressionsanalysen. ${ }^{10}$

\section{a) Dimension: Kompetenzerwerb}

Die Dimension Kompetenzerwerb soll entsprechend des Forschungsstandes über die drei Kriterien Fakten- und Sachwissen, Zunahme von Interesse und informeller Lernerfolg untersucht werden.

\section{(1) Erwerb von Fakten- und Sachwissen}

Die Auswertung zeigt, dass die Teilnehmer der Bürgerkonferenz häufig angeben, allgemein mehr über das Thema Energie erfahren zu haben. Diese Zustimmung nimmt zwar zwei Monate nach der Bürgerkonferenz wieder signifikant ab, aber der Unterschied ist nur gering. Vergleichsweise gering ist die Zustimmung auf die

10 Für die Mittelwertvergleiche greifen wir auf nichtparametrische Tests zurück, weil diese auch bei kleinen Fallzahlen und ohne die Notwendigkeit einer Normalverteilung angewendet werden dürfen. Als signifikant gelten alle Ergebnisse mit $p<=0,05$. Zur statistischen Absicherung von gruppenspezifischen Mittelwertunterschieden werden zusätzlich Regressionsanalysen durchgeführt. Zunächst werden bivariate Regressionsmodelle gerechnet; wenn diese Ergebnisse sich vom Mittelwertvergleich unterscheiden, wird anschließend mit Hilfe des sequentiellen Modells der Einfluss der anderen Variablen getestet. Angegeben werden bei den relevanten Regressionsanalysen: $\mathrm{R}^{2}$ (Bestimmtheitsmaß/Anteil der durch die Regression erklärten Varianz), b (Regressionskoeffizient/ Steigung der Regression), SE (Standardfehler), p (Signifikanzniveau) und a (Konstante bzw. Ordinatenabschnitt der Regression). Durch die multivariaten Analysen kann ermittelt werden, ob die Effekte, die über die bivariaten Analysen erzielt werden, unter Kontrolle der anderen unabhängigen Variablen bestehen bleiben. Allerdings sind die Residuen im multivariaten Modell nicht immer normalverteilt. Enge Grenzen von +1 und -1 für die Schiefe der Residuen, wie sie beispielsweise Urban/Mayerl (2008: 196) empfehlen, können mit den vorliegenden Daten häufig nicht eingehalten werden. Wir nehmen daher eine Normalverteilung an, wenn die Werte für die Schiefe der Residuen zwischen -3.21 und +3.21 liegen (vgl. Curran/West/Finch 1996). Es zeigt sich, dass auf der inhaltlichen Ebene keine nennenswerten Unterschiede zwischen den Mittelwertvergleichen und den Regressionsanalysen vorzufinden sind. Die Grundaussage dieses Artikels wird bestätigt: Die Bildung hat im Vergleich zum Geschlecht und dem Alter der Befragten den stärksten Effekt. Auffälligkeiten bzw. Abweichungen werden bei den betreffenden Variablen kommentiert.

Aus Gründen der besseren Lesbarkeit haben wir in den Tabellen und Fußnoten die Werte auf zwei Nachkommastellen gerundet. 
Marlen Niederberger, Verena Keierleber und Isabel Schneider

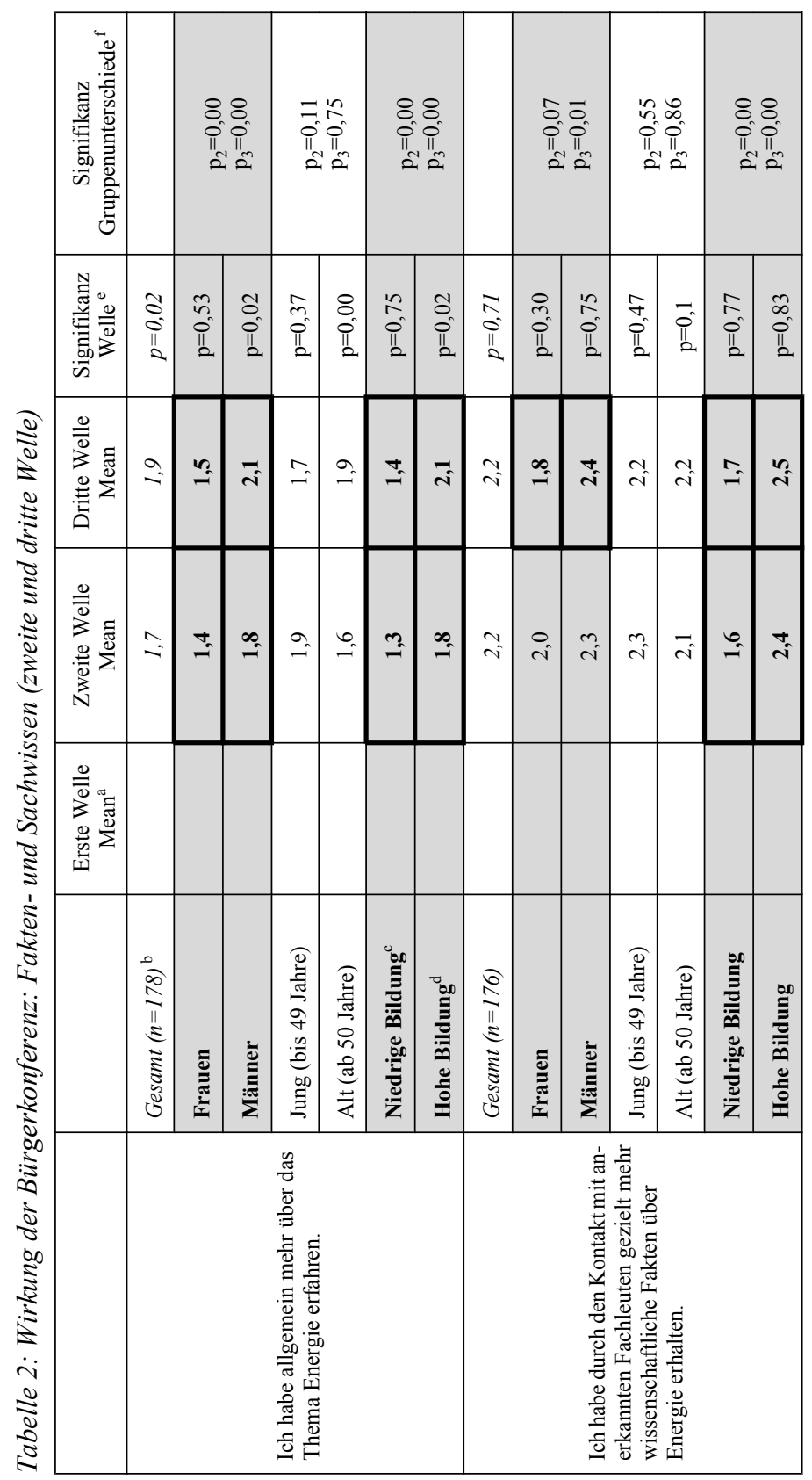


Kompetenzerwerb und Meinungsbildung in Bürgerkonferenzen

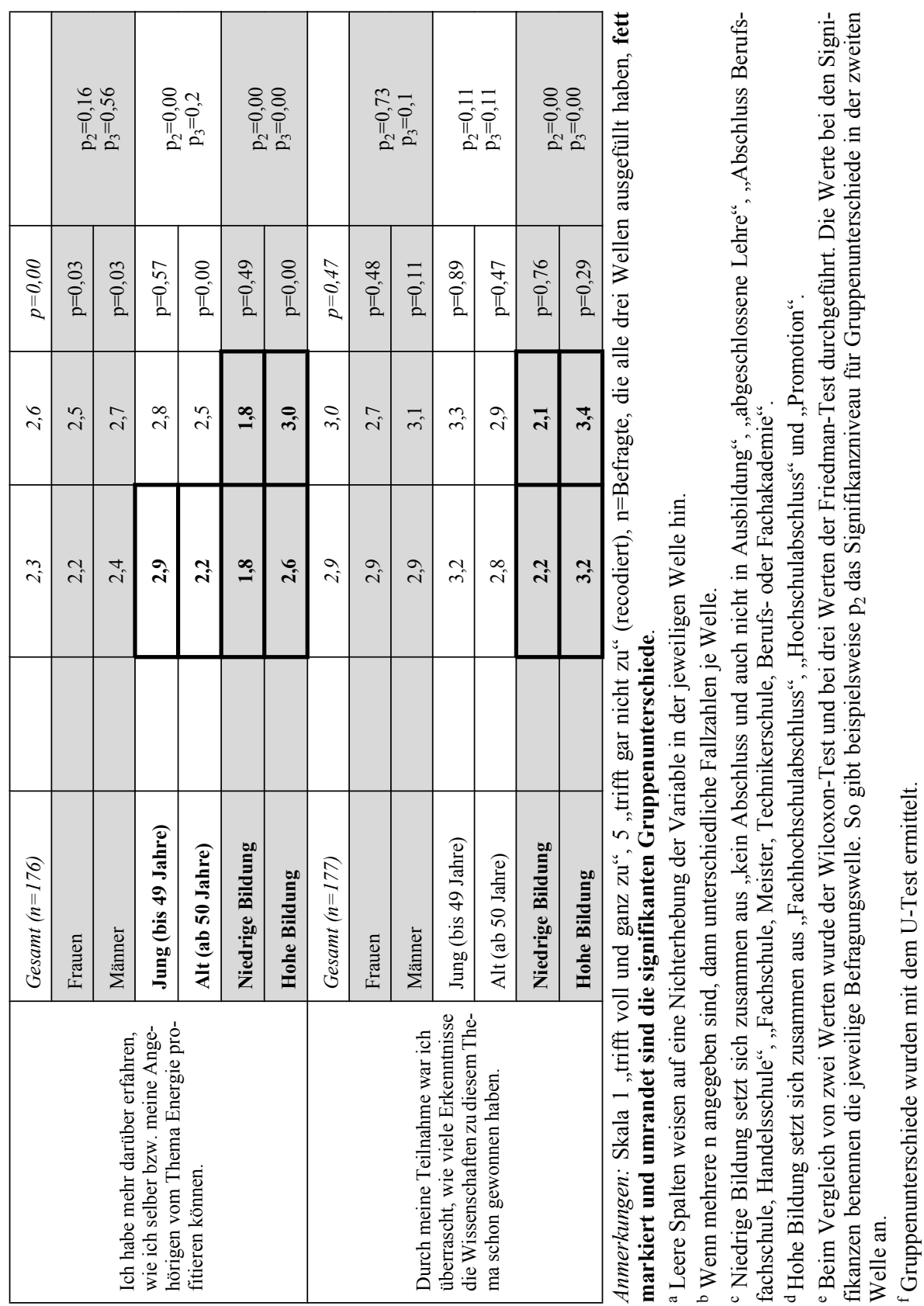


Frage, ob man mehr darüber erfahren hat, wie man selbst bzw. Angehörige vom Thema Energie profitieren können (siehe Tab. 2).

Die Betrachtung der Gruppenunterschiede zeigt einige Besonderheiten: Frauen geben gemessen am Durchschnitt signifikant stärker als Männer an, neues Wissen erlangt zu haben. Doch zwei der gefundenen signifikanten Geschlechtsunterschiede sind durch die multivariaten Regressionsanalysen zu relativieren bzw. zu konkretisieren:

- Bei der Variable „Ich habe allgemein mehr über Energie erfahren“(Welle 2) wird durch Hinzunahme der Variable Bildung der Effekt der Variable Geschlecht nicht signifikant. ${ }^{11}$ Nimmt man auch noch Alter hinzu, dann sind Geschlecht und Alter signifikant. ${ }^{12}$ Da dieses Modell insgesamt die höchste Erklärungskraft hat, ist davon auszugehen, dass Geschlecht und Alter hier die zentralen Variablen sind. Das heißt, vor allem Frauen und jüngere Personen lernen allgemein mehr über Energie.

- Bei der Variable „Ich habe durch den Kontakt mit anerkannten Fachleuten gezielt mehr wissenschaftliche Fakten über Energie erhalten" (Welle 3) erweist sich Bildung als die zentrale Variable. Nimmt man diese hinzu, verschwindet der Geschlechtseffekt. ${ }^{13}$ Das kann daran liegen, dass in dem vorliegenden Fall der Anteil der niedrig gebildeten Personen bei den Frauen etwas höher ist als bei den Männern, und die Frauen eher angeben, Neues über das Thema Energie gelernt zu haben. Das Alter hat hier keinen Einfluss. ${ }^{14}$

Bei der Bildung zeigt sich, dass Personen mit niedriger formaler Bildung signifikant eher glauben, mehr gelernt zu haben, als Personen mit hoher formaler Bildung. Des Weiteren geben Personen mit niedriger formaler Bildung signifikant stärker an, durch den Kontakt mit anerkannten Fachleuten mehr wissenschaftliche Fakten zum Thema erhalten zu haben, als Personen mit hoher formaler Bildung. Auch den Bezug $\mathrm{zu}$ sich und Angehörigen können Personen mit niedriger formaler Bildung eher herstellen. Diese Personengruppe ist eher überrascht, wie viele Erkenntnisse die Wissenschaft zum Thema Energie bereits gewonnen hat. Die Ergebnisse der Regressionsanalysen bestätigen den ermittelten Bildungseffekt. Die Bildung hat bei

11 Regressionsmodell mit Geschlecht und Bildung: $\mathrm{R}^{2}=0,06$; Geschlecht $b=0,34(\mathrm{SE}=0,02) \mathrm{p}=0,05$; Bildung $b=0,42(\mathrm{SE}=0,18) \mathrm{p}=0,02 ; \mathrm{a}=1,16 \mathrm{p}=0,00$.

12 Regressionsmodell mit Geschlecht, Bildung und Alter: $\mathrm{R}^{2}=0,09$; Geschlecht $b=0,43(\mathrm{SE}=0,18)$ $\mathrm{p}=0,02$; Bildung $\mathrm{b}=0,34(\mathrm{SE}=0,18) \mathrm{p}=0,06$; Alter $\mathrm{b}=-0,42(\mathrm{SE}=0,2) \mathrm{p}=0,04 ; \mathrm{a}=1,47 \mathrm{p}=0,00$.

13 Regressionsmodell mit Geschlecht und Bildung: $R^{2}=0,1$; Geschlecht $b=0,42(S E=0,22) p=0,06$; Bildung $b=0,76(\mathrm{SE}=0,23) \mathrm{p}=0,00 ; a=1,43 \mathrm{p}=0,00$.

14 Regressionsmodell mit Geschlecht, Bildung und Alter: $\mathrm{R}^{2}=0,1$; Geschlecht $b=0,44(\mathrm{SE}=0,23)$ $\mathrm{p}=0,06$; Bildung $\mathrm{b}=0,75(\mathrm{SE}=0,23) \mathrm{p}=0,00$; Alter $\mathrm{b}=-0,07(\mathrm{SE}=0,25) \mathrm{p}=0,78 ; \mathrm{a}=1,49 \mathrm{p}=0,00$. 
allen Variablen zum Thema Fakten- und Sachwissen einen signifikanten Effekt und stellt sich damit als zentrale Variable heraus.

Beim Alter zeigt sich ein signifikanter Mittelwertunterschied bei der Variable „Ich habe mehr darüber erfahren, wie ich selber bzw. meine Angehörigen vom Thema Energie profitieren können“" (Welle 2). Diesem Item stimmen ältere Personen stärker zu als die Jüngeren. Der Effekt bestätigt sich bei den Regressionsanalysen. Alter und Bildung haben einen signifikanten Einfluss. ${ }^{15}$

\section{(2) Zunahme an Interesse}

Viele Teilnehmer geben sowohl unmittelbar nach der Veranstaltung als auch zwei Monate später an, zukünftig die Diskussionen zum Thema Energie intensiver verfolgen zu wollen. Außerdem möchten sie sich bei Energiefragen gesellschaftlich mehr engagieren. Damit kann unterstellt werden, dass das Interesse an dem Thema und die intrinsische Motivation, sich damit auseinanderzusetzen, nachhaltig zugenommen haben. Diese Angaben ändern sich auch zwei Monate nach der Veranstaltung nicht signifikant (vgl. Tab. 3).

Das Interesse der Personen mit niedriger formaler Bildung ist sowohl zum Messzeitpunkt 2 als auch zum Messzeitpunkt 3 bei beiden Items signifikant höher als das Interesse von Personen mit höherer Bildung. Sie möchten das Thema zukünftig mehr verfolgen und sich auch verstärkt gesellschaftlich engagieren. Die Regressionsanalysen bestätigen den signifikanten Bildungseffekt.

Die Untersuchungen der Gruppenunterschiede hinsichtlich des Geschlechts und des Alters bringen keine signifikanten Ergebnisse hervor. Der einzige signifikante Unterschied bei der Variable Alter, der mit dem Mittelwertvergleich bezüglich des Engagements ermittelt wurde, wird in den Regressionsanalysen durch Bildung überlagert. ${ }^{16}$ Zwar ist Alter im Modell mit allen drei Variablen auch knapp signifikant, aber der Bildungseffekt ist stärker.

15 Regressionsmodell mit Bildung und Alter: $\mathrm{R}^{2}=0,15$; Bildung $\mathrm{b}=0,79(\mathrm{SE}=0,22) \mathrm{p}=0,00$; Alter $\mathrm{b}=-0,74(\mathrm{SE}=0,23) \mathrm{p}=0,00 ; \mathrm{a}=2,33 \mathrm{p}=0,00$.

Regressionsmodell mit Geschlecht, Bildung und Alter: $\mathrm{R}^{2}=0,15$; Geschlecht $\mathrm{b}=0,14$ (SE 0,22) $\mathrm{p}=0,52$; Bildung $\mathrm{b}=0,76(\mathrm{SE}=0,22) \mathrm{p}=0,00$; Alter $\mathrm{b}=-0,77(\mathrm{SE}=0,24) \mathrm{p}=0,00 ; \mathrm{a}=2,33 \mathrm{p}=0,00$.

16 Regressionsmodell mit Alter und Bildung: $\mathrm{R}^{2}=0,05$; Alter $\left.\mathrm{b}=0,41 \quad \mathrm{SE}=0,22\right) \mathrm{p}=0,06$; Bildung $\mathrm{b}=0,48(\mathrm{SE}=0,20) \mathrm{p}=0,02 ; \mathrm{a}=1,41 \mathrm{p}=0,00$.

Regressionsmodell mit Alter, Geschlecht und Bildung: $\mathrm{R}^{2}=0,05$; Alter $\mathrm{b}=0,44(\mathrm{SE}=0,22) \mathrm{p}=0,05$; Bildung $b=0,5(\mathrm{SE}=0,20) \mathrm{p}=0,02$; Geschlecht $\mathrm{b}=-0,11(\mathrm{SE}=0,20) \mathrm{p}=0,56 ; \mathrm{a}=1,44 \mathrm{p}=0,00$. 
Marlen Niederberger, Verena Keierleber und Isabel Schneider

Tabelle 3: Wirkung der Bürgerkonferenz: Interesse und Eigeninitiative (zweite und dritte Welle)

\begin{tabular}{|c|c|c|c|c|c|c|}
\hline & & $\begin{array}{l}\text { Erste } \\
\text { Welle } \\
\text { Mean }^{\mathrm{a}}\end{array}$ & $\begin{array}{l}\text { Zweite } \\
\text { Welle } \\
\text { Mean }\end{array}$ & $\begin{array}{l}\text { Dritte } \\
\text { Welle } \\
\text { Mean }\end{array}$ & $\begin{array}{l}\text { Signifikanz } \\
\text { Welle }^{\mathrm{e}}\end{array}$ & $\begin{array}{l}\text { Signifikanz } \\
\text { Gruppenun- }_{\text {terschiede }^{f}}\end{array}$ \\
\hline \multirow{7}{*}{$\begin{array}{l}\text { Durch meine Teilnahme } \\
\text { möchte ich zukünftig die } \\
\text { politischen Diskussionen } \\
\text { zum Thema Energie in- } \\
\text { tensiver verfolgen. }\end{array}$} & $\begin{array}{l}\text { Gesamt } \\
(n=177)\end{array}$ & & 1,6 & 1,7 & $p=0,81$ & \\
\hline & Frauen & & 1,5 & 1,6 & $\mathrm{p}=0,27$ & \multirow{2}{*}{$\begin{array}{l}\mathrm{p}_{2}=0,19 \\
\mathrm{p}_{3}=0,99\end{array}$} \\
\hline & Männer & & 1,7 & 1,7 & $\mathrm{p}=0,74$ & \\
\hline & $\begin{array}{l}\text { Jung } \\
\text { (bis 49 Jahre) }\end{array}$ & & 1,7 & 1,8 & $\mathrm{p}=0,58$ & \multirow{2}{*}{$\begin{array}{l}\mathrm{p}_{2}=0,29 \\
\mathrm{p}_{3}=0,16\end{array}$} \\
\hline & $\begin{array}{l}\text { Alt } \\
\text { (ab 50 Jahre) }\end{array}$ & & 1,6 & 1,6 & $\mathrm{p}=0,93$ & \\
\hline & $\begin{array}{l}\text { Niedrige } \\
\text { Bildung }\end{array}$ & & 1,2 & 1,3 & $\mathrm{p}=0,33$ & \multirow{2}{*}{$\begin{array}{l}\mathrm{p}_{2}=0,00 \\
\mathrm{p}_{3}=0,00\end{array}$} \\
\hline & Hohe Bildung & & 1,9 & 1,8 & $\mathrm{p}=0,72$ & \\
\hline \multirow{7}{*}{$\begin{array}{l}\text { Durch meine Teilnahme } \\
\text { möchte ich mich bei En- } \\
\text { ergiefragen gesellschaft- } \\
\text { lich mehr engagieren. }\end{array}$} & $\begin{array}{l}\text { Gesamt } \\
(n=177)\end{array}$ & & 1,9 & 2,1 & $p=0,12$ & \\
\hline & Frauen & & 2,0 & 2,0 & $\mathrm{p}=0,96$ & \multirow{2}{*}{$\begin{array}{l}\mathrm{p}_{2}=0,71 \\
\mathrm{p}_{3}=0,47\end{array}$} \\
\hline & Männer & & 1,9 & 2,1 & $\mathrm{p}=0,05$ & \\
\hline & $\begin{array}{l}\text { Jung } \\
\text { (bis 49 Jahre) }\end{array}$ & & 1,8 & 1,8 & $\mathrm{p}=0,74$ & \multirow{2}{*}{$\begin{array}{l}\mathrm{p}_{2}=0,25 \\
\mathrm{p}_{3}=0,02\end{array}$} \\
\hline & $\begin{array}{l}\text { Alt } \\
\text { (ab 50 Jahre) }\end{array}$ & & 2,0 & 2,2 & $\mathrm{p}=0,08$ & \\
\hline & $\begin{array}{l}\text { Niedrige } \\
\text { Bildung }\end{array}$ & & 1,6 & 1,8 & $\mathrm{p}=0,19$ & \multirow{2}{*}{$\begin{array}{l}\mathrm{p}_{2}=0,02 \\
\mathrm{p}_{3}=0,08\end{array}$} \\
\hline & Hohe Bildung & & 2,1 & 2,2 & $\mathrm{p}=0,23$ & \\
\hline
\end{tabular}

Anmerkungen: Siehe Tabelle 2.

\section{(3) Informeller Lernerfolg}

Die Aneignung sozialer, demokratischer und wissenschaftlicher Kompetenzen kann mit dem vorhandenen Datenmaterial nur bedingt untersucht werden. Die entsprechenden abgefragten Items beziehen sich auf soziale und kommunikative Aspekte sowie auf die Selbsteinschätzung, an derartigen Veranstaltungen kompetent teilnehmen zu können.

Ein Großteil der Teilnehmer gibt an, auf der Veranstaltung neue Menschen kennengelernt zu haben. Unklar bleibt allerdings, ob der Kontakt mit anderen Teilneh- 
mern nach der Veranstaltung weiter besteht. Allerdings nimmt die Zustimmung auf diese Frage in der dritten Welle signifikant ab, was darauf hindeutet, dass zumindest nicht alle den Kontakt halten konnten.

Die Zustimmung auf die Frage, ob man durch die Teilnahme gelernt habe, wie man im Dialog mit anderen zu fundierten Urteilen kommen kann, liegt im mittleren Bereich. Zwei Monate nach der Veranstaltung ist die durchschnittliche Zustimmung signifikant höher, das heißt die Bürger glauben stärker an diesen Lernerfolg.

Im unmittelbaren Vergleich vor und nach der Bürgerkonferenz geben die Befragten signifikant häufiger an, über genügend Erfahrung zu verfügen, um aktiv an Veranstaltungen teilzunehmen, bei denen Bürger an gesellschaftlichen Entscheidungen beteiligt werden. Der Vergleich der Messzeitpunkte 2 und 3 liefert keine signifikanten Unterschiede. Die Effekte sind deshalb auf die Teilnahme an der Bürgerkonferenz zurückzuführen.

Auch beim informellen Lernen zeigen sich bei der Bildung signifikante Gruppenunterschiede. In der zweiten und dritten Welle geben Personen, die mindestens einen Fachhochschulabschluss haben, signifikant häufiger an, über genügend Erfahrungen zu verfügen, um an derartigen Verfahren teilzunehmen, als Personen mit niedriger formaler Bildung. Sie schätzen ihre Erfahrung also eher als ausreichend für die Teilnahme an einer Bürgerkonferenz ein, geben aber gleichzeitig signifikant weniger an, durch den Dialog gelernt zu haben, wie man mit anderen zu einem fundierten Urteil kommt. Die Regressionsanalysen bestätigen die signifikanten $\mathrm{Zu}-$ sammenhänge zwischen Bildung und dem informellen Lernerfolg. Insgesamt ist hier Bildung die Variable mit dem stärksten Effekt. Auch der Altersunterschied bleibt bei der Variable „Ich habe genug Erfahrung, um aktiv an Veranstaltungen teilzunehmen, bei denen Bürger an gesellschaftlichen Entscheidungen beteiligt werden" (Welle 3) neben Bildung in der Regressionsanalyse signifikant (vgl. Tab. 4). ${ }^{17}$

17 Regressionsmodell mit Alter, Geschlecht und Bildung: $\mathrm{R}^{2}=0,08$; Alter $\mathrm{b}=0,39(\mathrm{SE}=0,19) \mathrm{p}=0,04$; Bildung $b=-0,35(\mathrm{SE}=0,17) \mathrm{p}=0,04$; Geschlecht $\mathrm{b}=-0,30(\mathrm{SE}=0,17) \mathrm{p}=0,07 ; \mathrm{a}=1,85 \mathrm{p}=0,00$. 
Marlen Niederberger, Verena Keierleber und Isabel Schneider

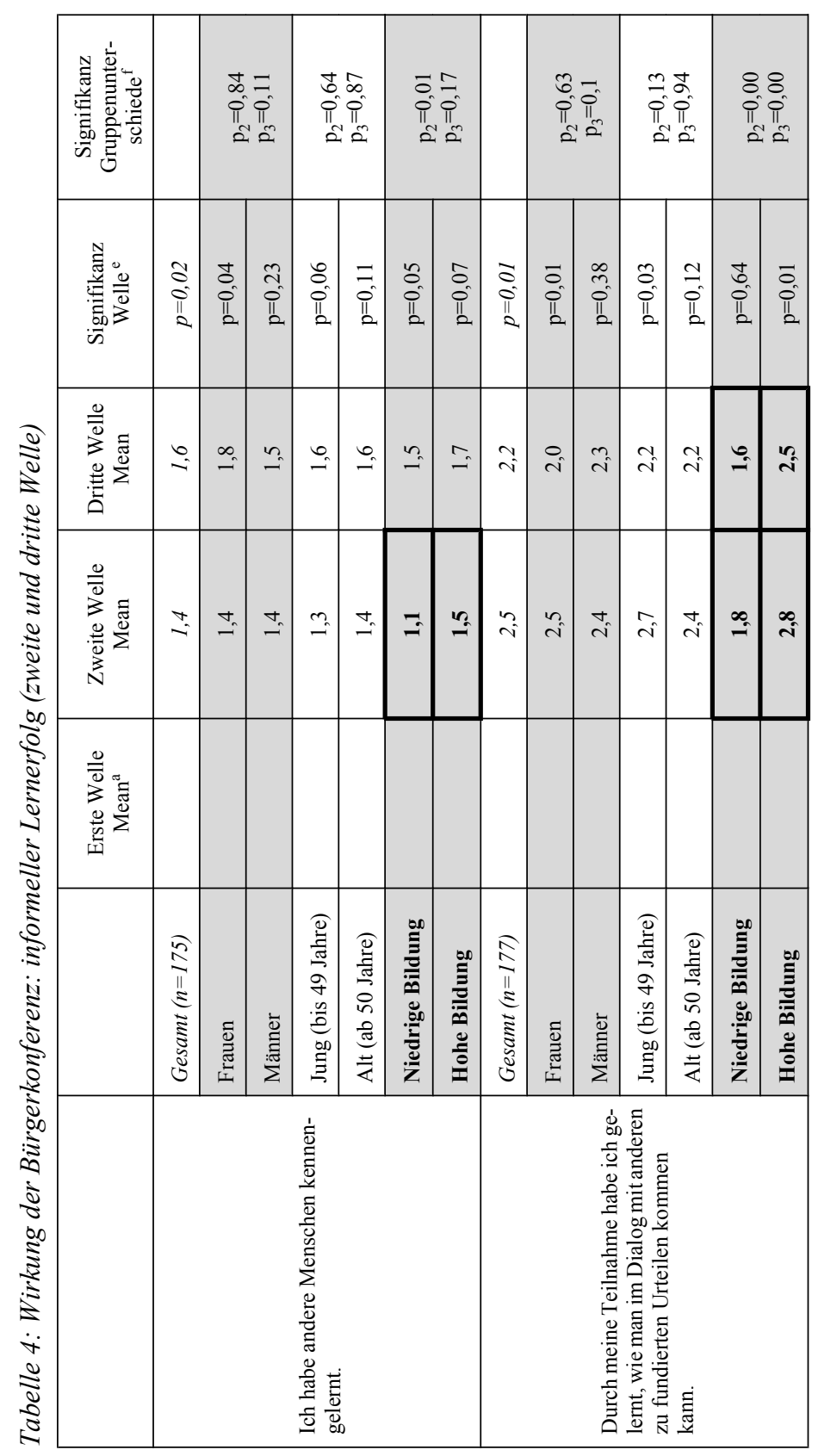


Kompetenzerwerb und Meinungsbildung in Bürgerkonferenzen

\begin{tabular}{|c|c|c|c|c|c|c|}
\hline & \multicolumn{2}{|c|}{ 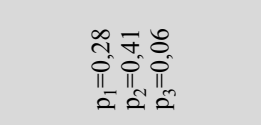 } & \multicolumn{2}{|c|}{ 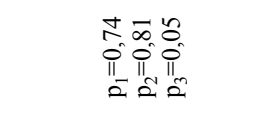 } & \multicolumn{2}{|c|}{ 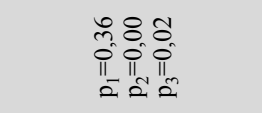 } \\
\hline 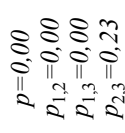 & 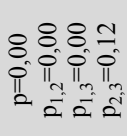 & 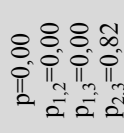 & 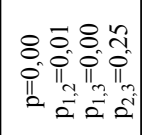 & 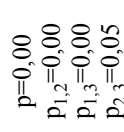 & 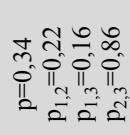 & 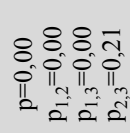 \\
\hline$\approx$ & $\stackrel{\circ}{i}$ & $\stackrel{6}{-}$ & $\cong$ & $\stackrel{\infty}{\rightarrow}$ & $\tilde{\sim}$ & $\stackrel{0}{=}$ \\
\hline$=$ & $\approx$ & $\stackrel{0}{-}$ & $\stackrel{0}{-}$ & $\approx$ & $\bar{i}$ & 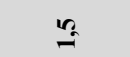 \\
\hline $\bar{v}$ & $\hat{i}$ & $\stackrel{\circ}{i}$ & î & $\vec{i}$ & तै & $\stackrel{\circ}{i}$ \\
\hline 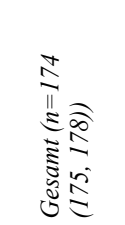 & Е & 离 & 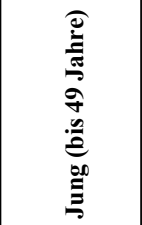 & 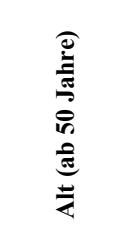 & 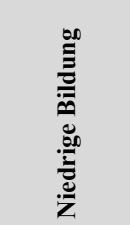 & 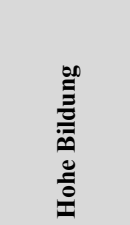 \\
\hline & & & 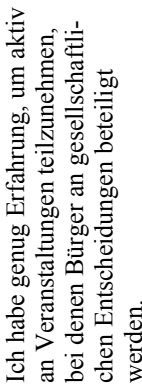 & & & \\
\hline
\end{tabular}




\section{(4) Fazit Kompetenzerwerb}

Im Ergebnis zeigt sich ein Kompetenzerwerb bei allen drei Kriterien.

- Das Sach- und Faktenwissen über Energie hat nach eigener Einschätzung der Bürger nach der Veranstaltung zugenommen. Im Vergleich nimmt dabei das thematische Wissen über Energie stärker zu als das Wissen durch den Kontakt mit anerkannten Fachleuten oder in Bezug auf Angehörige.

- Auch das Interesse am Thema Energie nimmt nach der Veranstaltung zu. Die Teilnehmer möchten das Thema mehr verfolgen und sich zukünftig stärker zu diesem Thema engagieren. Diese Einschätzung ändert sich auch zwei Monate nach der Veranstaltung nicht signifikant.

- Lernerfolge zeigen sich bei der kommunikativen Kompetenz. Die Bürger haben zum Großteil gelernt, wie man in der Diskussion mit anderen zu einem gemeinsamen Urteil kommen kann. Dieser Effekt verstärkt sich zwei Monate nach der Veranstaltung sogar signifikant. Ein weiterer informeller Lernerfolg betrifft die subjektive Einschätzung, genug Erfahrung zu haben, um aktiv an Bürgerbeteiligungsverfahren teilzunehmen. Der Vergleich der Ergebnisse vor und nach der Veranstaltung liefert einen signifikanten Anstieg in der Zustimmung zu dieser Frage.

- Der Vergleich zwischen Personen mit niedriger formaler Bildung und höherer formaler Bildung zeigt einige signifikante Unterschiede. Personen mit hoher Bildung geben nach der Veranstaltung eher an, über genügend Erfahrungen für die Teilnahme an solchen Veranstaltungen zu verfügen. Personen mit niedriger Bildung haben nach eigener Einschätzung mehr Sachwissen erlangt. Auch das Interesse und die Motivation, sich in diesem Thema gesellschaftlich zu engagieren, haben bei dieser Gruppe stärker zugenommen.

- Beim Geschlecht zeigen sich vor allem Effekte beim Wissen über Energie. Frauen schätzen ihren Lernzuwachs signifikant höher ein als Männer.

- Beim Alter zeigen sich keine gravierenden Unterschiede zwischen jüngeren und älteren Teilnehmern. Allerdings können die älteren Teilnehmer mit Blick auf das Thema eher einen Bezug zu sich und ihren Angehörigen herstellen.

\section{b) Dimension: Meinungsbildung bzw. -änderung}

Veränderungen in der Dimension Meinungsbildung oder -änderung können mit dem Datenmaterial anhand von drei Aspekten untersucht werden: Meinung gegenüber Wissenschaftlern und Politikern, Meinung zur Bürgerbeteiligung in Deutschland und der Standpunkt zum Thema Energie. 


\section{(1) Meinung über Wissenschaftler und Politiker}

Die Zustimmungen zu den Fragen, ob die Bürger durch ihre Teilnahme den Eindruck bekommen haben, dass Wissenschaftler bzw. Politiker an Gesprächen mit Bürgern wirklich interessiert sind, liegen im mittleren Bereich. Allerdings nimmt diese Meinung bezüglich der Politiker zwei Monate nach der Veranstaltung signifikant ab. Dass sich durch die Teilnahme die Meinung der Bürger über Wissenschaftler und Politiker verändert hat, geht aus den Ergebnissen nicht hervor.

Die Analyse der Gruppenunterschiede zeigt nur bei der Variable Bildung Auffälligkeiten. Stärkere Effekte zeigen sich bei den Bürgern mit niedriger formaler Bildung. Sie haben nach der Bürgerkonferenz eher den Eindruck, dass Wissenschaftler und Politiker an der Meinung der Bürger interessiert sind und haben ihre Meinung gegenüber diesen Personengruppen stärker geändert als Personen mit hoher formaler Bildung. Die Regressionsanalysen bestätigen den signifikanten Bildungseffekt bei allen Variablen (vgl. Tab. 5).

\section{(2) Bürgerbeteiligung in Deutschland}

Drei Items zum Thema Bürgerbeteiligung in Deutschland wurden in allen drei Wellen untersucht. Dabei geht es um die Fragen, ob Bürger in Deutschland nach Ansicht der Befragten gesellschaftliche Entscheidungen beeinflussen können, ob Bürgerbeteiligung die Qualität politischer Entscheidungen verbessert und ob eher Experten die Entscheidung über komplexe wissenschaftlich-technische Themen treffen sollten. Mit diesen Items wird die Meinungsänderung auf politischer Ebene gemessen (Tab. 6).

Nach der Veranstaltung stimmen die Befragten im Schnitt stärker zu, dass in Deutschland Bürger kaum Möglichkeiten haben, gesellschaftliche Entscheidungen mit zu beeinflussen. Dieses Ergebnis ist allerdings nicht signifikant.

Das Item, ob Bürgerbeteiligung im Entscheidungsprozess die Qualität politischer Entscheidungen verbessert, zeigt signifikante Veränderungen. Unmittelbar nach der Veranstaltung stimmen die Teilnehmer dieser Aussage signifikant mehr zu. Nach zwei Monaten ändert sich dies aber wieder und der Ursprungswert wird angenommen.

Auf die Frage, ob Entscheidungen zu komplexen wissenschaftlich-technischen Themen von Experten getroffen werden sollten, sind die meisten Teilnehmer ambivalent eingestellt. Nach der Veranstaltung lehnen sie dieses Item stärker ab. Dieser Effekt ist bei Welle $1 \mathrm{im}$ Vergleich zu Welle 2 und 3 signifikant. 
Marlen Niederberger, Verena Keierleber und Isabel Schneider

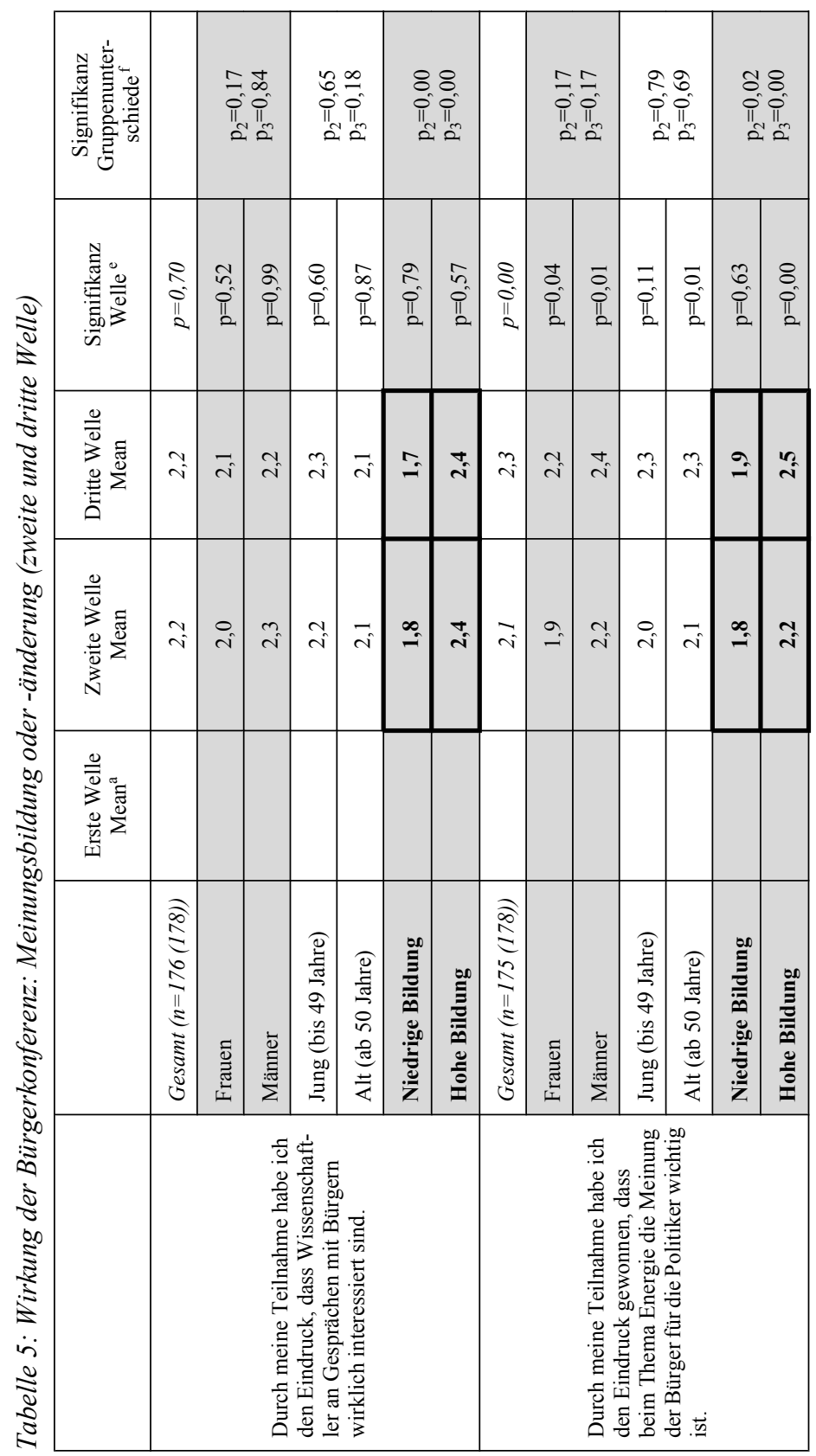




\begin{tabular}{|c|c|c|c|c|c|c|c|c|c|c|c|c|c|}
\hline & \multicolumn{2}{|c|}{$\begin{array}{l}\tilde{N} \approx \\
\tilde{N} \|^{\circ} \\
\tilde{e}=\end{array}$} & \multicolumn{2}{|c|}{$\begin{array}{l}\bar{n} \tilde{v} \\
\tilde{0} \tilde{o} \\
\left\|_{N}\right\|_{m} \\
\tilde{=}=\end{array}$} & \multicolumn{2}{|c|}{ 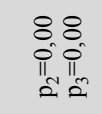 } & \multicolumn{3}{|c|}{ 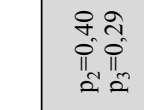 } & \multicolumn{2}{|c|}{ 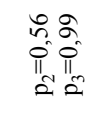 } & \multicolumn{2}{|c|}{ 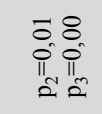 } \\
\hline $\begin{array}{l}\hat{n} \\
\hat{0} \\
2\end{array}$ & 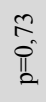 & $\begin{array}{l}\text { in } \\
0 \\
0 \\
0\end{array}$ & 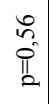 & $\begin{array}{l}n \\
\cdots \\
\hat{l}_{0} \\
2\end{array}$ & $\begin{array}{l}\vec{n} \\
0 \\
0 \\
2\end{array}$ & 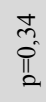 & $\begin{array}{l}\infty \\
m \\
\prod_{2}^{2} \\
2\end{array}$ & $\begin{array}{l}\vec{F} \\
\stackrel{0}{0} \\
\text { in }\end{array}$ & $\begin{array}{l}\hat{3} \\
0 \\
0 \\
0\end{array}$ & $\begin{array}{l}\vec{z} \\
\overrightarrow{0} \\
\underline{0}\end{array}$ & $\begin{array}{l}+ \\
\infty \\
0 \\
0 \\
0\end{array}$ & $\begin{array}{l}0 \\
0 \\
0 \\
i \\
0\end{array}$ & $\begin{array}{l}\infty \\
0 \\
0 \\
0\end{array}$ \\
\hline $\begin{array}{l}0 \\
\dot{n}\end{array}$ & $\ddot{n}$ & $\stackrel{0}{m}$ & $\stackrel{\infty}{m}$ & $\stackrel{n}{m}$ & $\stackrel{n}{*}$ & $\stackrel{8}{*}$ & $\begin{array}{l}\infty \\
m^{\prime}\end{array}$ & $\stackrel{0}{m}$ & ले & $\stackrel{\infty}{m}$ & $\stackrel{\infty}{m}$ & $\stackrel{\rho}{\rho}$ & $\bar{f}$ \\
\hline$\hat{m}$ & $\stackrel{\circ}{m}$ & $\stackrel{\infty}{m}$ & $\stackrel{0}{\circ}$ & $\stackrel{0}{m}$ & $\stackrel{\infty}{i}$ & F & $\stackrel{\infty}{m}$ & $\hat{m}$ & ले & $\stackrel{\circ}{+}$ & $\stackrel{\infty}{m}$ & $\stackrel{\oplus}{m}$ & $F$ \\
\hline 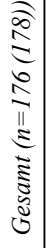 & 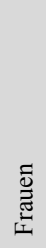 & $\begin{array}{l}\bar{\Xi} \\
\stackrel{\Xi}{\Sigma} \\
\sum\end{array}$ & 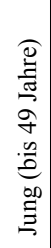 & 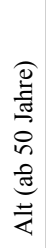 & 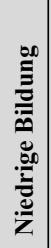 & 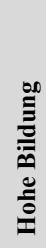 & 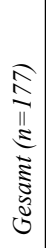 & 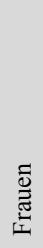 & 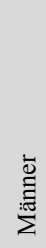 & 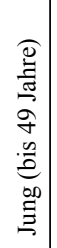 & 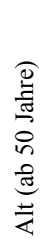 & 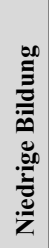 & 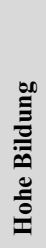 \\
\hline & & & 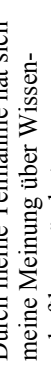 & & & & & & & 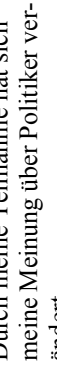 & & & \\
\hline
\end{tabular}


Marlen Niederberger, Verena Keierleber und Isabel Schneider

\begin{tabular}{|c|c|c|c|c|c|c|c|}
\hline 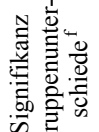 & & \multicolumn{2}{|c|}{ 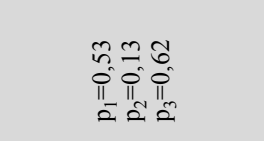 } & \multicolumn{2}{|c|}{ 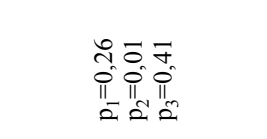 } & \multicolumn{2}{|c|}{ 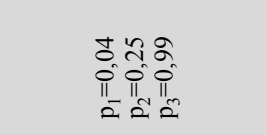 } \\
\hline 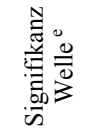 & 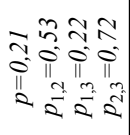 & 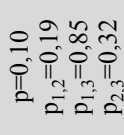 & 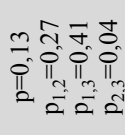 & 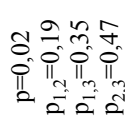 & 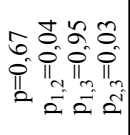 & 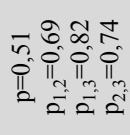 & 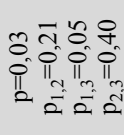 \\
\hline 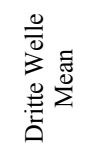 & $\begin{array}{l}n \\
\sim i\end{array}$ & $\underset{i}{0}$ & $\stackrel{n}{n}$ & $\begin{array}{l}6 \\
i\end{array}$ & $\stackrel{n}{i}$ & $\hat{n}$ & $\tilde{n}$ \\
\hline 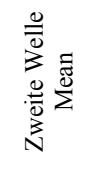 & $\begin{array}{l}b \\
\sim\end{array}$ & $\stackrel{\infty}{i}$ & $\stackrel{\nabla}{i}$ & $\bar{m}$ & $\stackrel{\Xi}{i}$ & $\stackrel{\vec{i}}{i}$ & $\stackrel{b}{i}$ \\
\hline 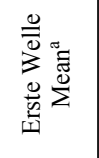 & $\hat{v}$ & $\stackrel{0}{i}$ & $\hat{i}$ & ते & $\stackrel{b}{i}$ & $\vec{i}$ & $\stackrel{\infty}{\sim}$ \\
\hline & 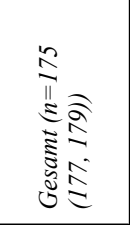 & 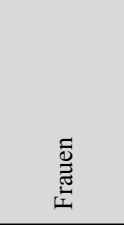 & 离 & 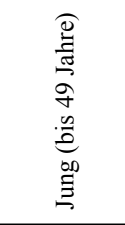 & 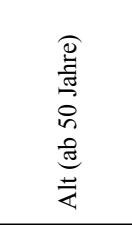 & 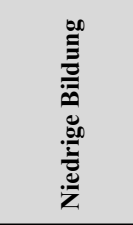 & 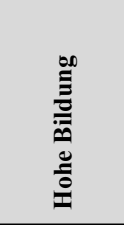 \\
\hline & & & & 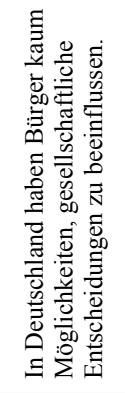 & & & \\
\hline
\end{tabular}


Kompetenzerwerb und Meinungsbildung in Bürgerkonferenzen

\begin{tabular}{|c|c|c|c|c|c|c|}
\hline & \multicolumn{2}{|c|}{ 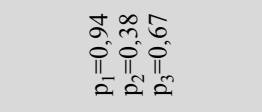 } & \multicolumn{2}{|c|}{ 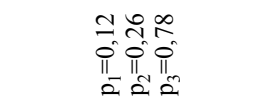 } & \multicolumn{2}{|c|}{ 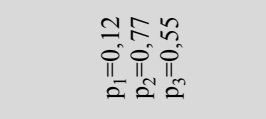 } \\
\hline 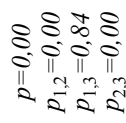 & 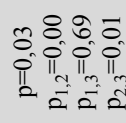 & 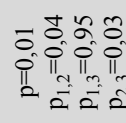 & 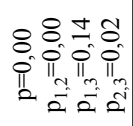 & 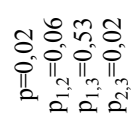 & 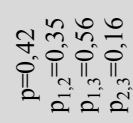 & 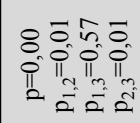 \\
\hline$\approx$ & $\stackrel{0}{-}$ & $\approx$ & $\stackrel{0}{-}$ & $=$ & $\stackrel{n}{=}$ & I \\
\hline$\underset{*}{*}$ & 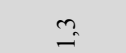 & $\stackrel{\Xi}{=}$ & 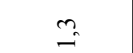 & $\underset{f}{+}$ & $\stackrel{ \pm}{=}$ & $\stackrel{\Xi}{=}$ \\
\hline$\hat{\therefore}$ & 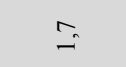 & $\stackrel{-}{\circ}$ & $\stackrel{\infty}{\rightarrow}$ & $\underset{-}{\circ}$ & $\stackrel{\Xi}{-}$ & $=$ \\
\hline 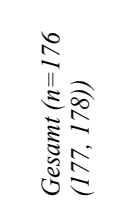 & $\begin{array}{l}\text { 品 } \\
\text { 胥 }\end{array}$ & 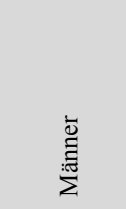 & 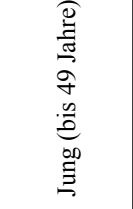 & 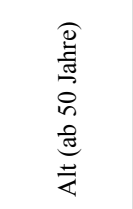 & 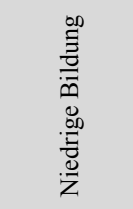 & 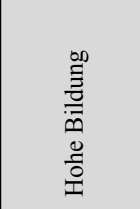 \\
\hline & & & 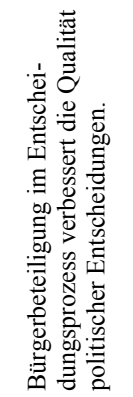 & & & \\
\hline
\end{tabular}


Marlen Niederberger, Verena Keierleber und Isabel Schneider

\begin{tabular}{|c|c|c|c|c|c|c|c|}
\hline 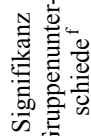 & & \multicolumn{2}{|c|}{ 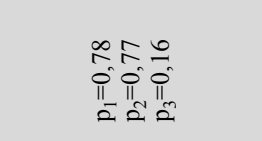 } & \multicolumn{2}{|c|}{ 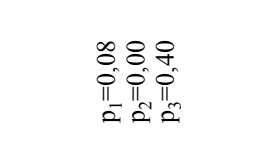 } & \multicolumn{2}{|c|}{ 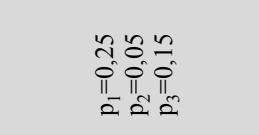 } \\
\hline 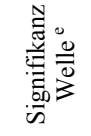 & 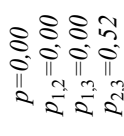 & 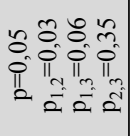 & 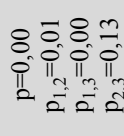 & 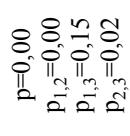 & 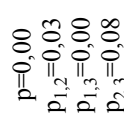 & 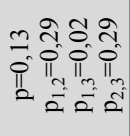 & 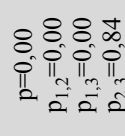 \\
\hline 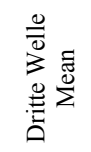 & $\stackrel{\infty}{m^{\prime}}$ & $\stackrel{6}{\circ}$ & ले & $\stackrel{\circ}{\rightarrow}$ & $\hat{m}$ & $\stackrel{6}{\sim}$ & $\stackrel{\circ}{*}$ \\
\hline 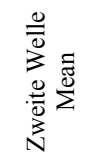 & $\hat{m}$ & $\stackrel{\infty}{\sim}$ & $\hat{m}$ & $\stackrel{+}{f}$ & $\stackrel{\varpi}{m}$ & $\stackrel{m}{m}$ & ले \\
\hline \multirow{3}{*}{ 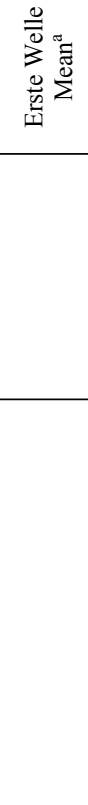 } & $\stackrel{m}{n}$ & $m^{m}$ & $\hat{m}^{m}$ & $\hat{m}$ & ñ & $\vec{m}$ & 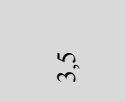 \\
\hline & 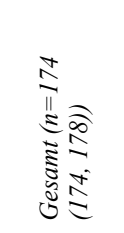 & 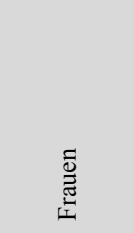 & $\begin{array}{l}\bar{\Xi} \\
\text { 离 } \\
\text { ¿ }\end{array}$ & 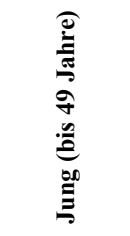 & 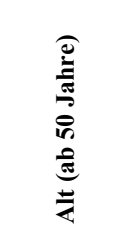 & 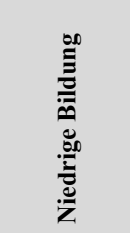 & 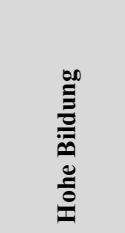 \\
\hline & \multicolumn{7}{|c|}{ 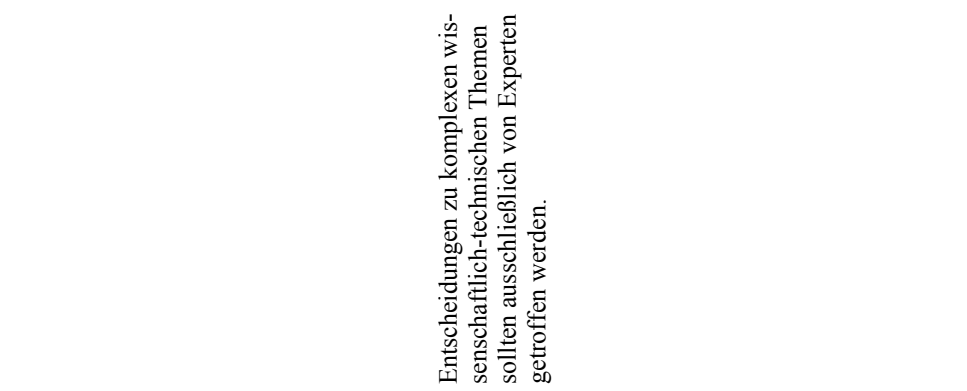 } \\
\hline
\end{tabular}


Signifikante Gruppenunterschiede sind bei diesen Fragen kaum zu finden. In der zweiten Befragungswelle stimmen ältere Teilnehmer eher zu, dass Bürger in Deutschland kaum Möglichkeiten haben, gesellschaftliche Entscheidungen mit zu beeinflussen und Entscheidungen zu komplexen technischen Fragestellungen eher den Experten überlassen werden sollten. Entscheidungen zu komplexen wissenschaftlich-technischen Themen sollten auch nach Ansicht von Personen mit niedriger formaler Bildung eher Experten überlassen werden. Die signifikanten Mittelwertunterschiede lassen sich durch die Regressionsanalysen bestätigen. Nur der Bildungseffekt in der zweiten Welle bezüglich der Entscheidung zu wissenschaftlich-technischen Fragestellungen wird bei der bivariaten Regressionsanalyse knapp nicht signifikant. Die multivariate Analyse deutet darauf hin, dass Alter hier die zentrale Variable ist. ${ }^{18}$

\section{(3) Standpunkt zum Thema}

Die Meinungsänderung wird auch zum Dialogthema Energie abgefragt. Dabei geht es zum einen um ein Globalurteil zum Thema „Steht man der Energiewende eher positiv oder negativ gegenüber?" und zum anderen um die Sicherheit des eigenen Standpunktes (vgl. Tab. 7).

Bei dem Globalurteil zeigt sich, dass zwei Monate nach der Bürgerkonferenz mehr Teilnehmer ein positives Urteil zum Atomausstieg vertreten als unmittelbar vor der Bürgerkonferenz. Vor der Veranstaltung gaben noch 22 Prozent der Teilnehmer an, dem Atomausstieg kritisch gegenüber zu stehen, beim Erhebungszeitpunkt zwei Monate danach betrug dieser Anteil 15 Prozent.

Tabelle 7: Wirkung der Veranstaltung: Meinungsbildung und-änderung (Globalurteil)

\begin{tabular}{|c|c|c|c|c|}
\hline & & Welle 1 & Welle 2 & Welle 3 \\
\hline \multirow[t]{2}{*}{$\begin{array}{l}\text { Alles in allem: Welche } \\
\text { Position würden Sie } \\
\text { eher vertreten? }\end{array}$} & Modus & $\begin{array}{l}\text { Positiv gegenüber } \\
\text { Atomausstieg: } \\
\text { Anteil der Nennung } \\
78 \%\end{array}$ & - & $\begin{array}{l}\text { Positiv gegenüber } \\
\text { Atomausstieg: } \\
\text { Anteil der Nennung } \\
85 \%\end{array}$ \\
\hline & $\mathrm{N}$ & 162 & - & 174 \\
\hline
\end{tabular}

Anmerkung: Skala: 1 „Person A (kritisch gegenüber Atomausstieg)“, 2 „Person B (positiv gegenüber Atomausstieg)“

Die Frage, ob die Befragten einen klaren Standpunkt zum Thema Energie haben, zeigt signifikante Veränderungen im Zeitverlauf der drei Wellen. Nach der Veran-

18 Regressionsmodell mit Geschlecht, Alter und Bildung: $\mathrm{R}^{2}=0,1$; Geschlecht $\mathrm{b}=0,14(\mathrm{SE}=0,25)$ $\mathrm{p}=0,58$; Bildung $\mathrm{b}=0,41(\mathrm{SE}=0,26) \mathrm{p}=0,12$; Alter $\mathrm{b}=-0,97(\mathrm{SE}=0,28) \mathrm{p}=0,00 ; \mathrm{a}=4,1 \mathrm{p}=0,00$. 
staltung vertreten die Teilnehmer signifikant häufiger einen klaren Standpunkt als zuvor. Hier deutet das Ergebnis auf eine signifikante Verfestigung der Meinung zur Zukunft der Energie hin (Tab. 8).

Signifikante Gruppenunterschiede sind anhand der Mittelwertvergleiche nur an einer Stelle zu erkennen. Direkt nach der Bürgerkonferenz geben Personen mit niedriger formaler Bildung signifikant stärker an, einen klaren Standpunkt zur Zukunft der Energie zu haben als Personen mit hoher formaler Bildung. Diesen Effekt bestätigt auch die bivariate Regression. ${ }^{19}$

Tabelle 8: Wirkung der Veranstaltung: Meinungsbildung und-änderung (Standpunkt)

\begin{tabular}{|c|c|c|c|c|c|c|}
\hline & & $\begin{array}{l}\text { Erste } \\
\text { Welle } \\
\text { Mean }^{\mathrm{a}}\end{array}$ & $\begin{array}{l}\text { Zweite } \\
\text { Welle } \\
\text { Mean }\end{array}$ & $\begin{array}{l}\text { Dritte } \\
\text { Welle } \\
\text { Mean }\end{array}$ & $\begin{array}{l}\text { Signifikanz } \\
\text { Welle }^{\mathrm{e}}\end{array}$ & $\begin{array}{c}\text { Signifikanz } \\
\text { Gruppenunterschiede }^{\mathrm{f}}\end{array}$ \\
\hline \multirow{7}{*}{$\begin{array}{l}\text { Ich habe zur Frage } \\
\text { der Zukunft der } \\
\text { Energie einen klaren } \\
\text { Standpunkt. }\end{array}$} & $\begin{array}{l}\text { Gesamt } \\
(n=175 \\
(176,178)\end{array}$ & 1,9 & 1,3 & 1,4 & $\begin{aligned} p & =0,00 \\
p_{1,2} & =0,00 \\
p_{1,3} & =0,00 \\
p_{2,3} & =0,08\end{aligned}$ & \\
\hline & Frauen & 2,0 & 1,4 & 1,5 & $\begin{aligned} \mathrm{p} & =0,00 \\
\mathrm{p}_{1,2} & =0,00 \\
\mathrm{p}_{1,3} & =0,00 \\
\mathrm{p}_{2,3} & =0,77\end{aligned}$ & \multirow{2}{*}{$\begin{array}{l}\mathrm{p}_{1}=0,30 \\
\mathrm{p}_{2}=0,68 \\
\mathrm{p}_{3}=0,54\end{array}$} \\
\hline & Männer & 1,8 & 1,3 & 1,4 & $\begin{aligned} \mathrm{p} & =0,00 \\
\mathrm{p}_{1,2} & =0,00 \\
\mathrm{p}_{1,3} & =0,00 \\
\mathrm{p}_{2,3} & =0,03\end{aligned}$ & \\
\hline & $\begin{array}{l}\text { Jung } \\
\text { (bis } 49 \text { Jahre) }\end{array}$ & 1,7 & 1,3 & 1,4 & $\begin{aligned} \mathrm{p} & =0,06 \\
\mathrm{p}_{1,2} & =0,01 \\
\mathrm{p}_{1,3} & =0,06 \\
\mathrm{p}_{2,3} & =0,52\end{aligned}$ & \multirow{2}{*}{$\begin{array}{l}\mathrm{p}_{1}=0,15 \\
\mathrm{p}_{2}=0,90 \\
\mathrm{p}_{3}=0,89\end{array}$} \\
\hline & $\begin{array}{l}\text { Alt } \\
\text { (ab 50 Jahre) }\end{array}$ & 2,0 & 1,3 & 1,4 & $\begin{aligned} \mathrm{p} & =0,00 \\
\mathrm{p}_{1,2} & =0,00 \\
\mathrm{p}_{1,3} & =0,00 \\
\mathrm{p}_{2,3} & =0,09\end{aligned}$ & \\
\hline & $\begin{array}{l}\text { Niedrige } \\
\text { Bildung }\end{array}$ & 1,9 & 1,1 & 1,4 & $\begin{array}{c}\mathrm{p}=0,00 \\
\mathrm{p}_{1,2}=0,00 \\
\mathrm{p}_{1,3}=0,00 \\
\mathrm{p}_{2,3}=0,01\end{array}$ & \multirow{2}{*}{$\begin{array}{l}\mathrm{p}_{1}=0,92 \\
\mathrm{p}_{2}=0,03 \\
\mathrm{p}_{3}=0,49\end{array}$} \\
\hline & $\begin{array}{l}\text { Hohe } \\
\text { Bildung }\end{array}$ & 1,9 & 1,4 & 1,4 & $\begin{array}{c}\mathrm{p}=0,00 \\
\mathrm{p}_{1,2}=0,00 \\
\mathrm{p}_{1,3}=0,00 \\
\mathrm{p}_{2,3}=0,46\end{array}$ & \\
\hline
\end{tabular}

Anmerkungen: Siehe Tabelle 2. 


\section{(4) Fazit Meinungsbildung und-änderung}

Im Ergebnis zeigen sich unterschiedliche Effekte auf die Meinungsbildung und -änderung.

- Die Meinung gegenüber Wissenschaftlern und Politikern hat sich bei vielen Bürgern nicht verändert. Bei den Items zur Bürgerbeteiligung zeigen sich jedoch Effekte: Sie glauben nach der Veranstaltung signifikant stärker, dass die Einbindung von Bürgern in politische Entscheidungsprozesse die Qualität dieser positiv beeinflusst. Sie glauben zudem weniger, dass Entscheidungen ausschließlich von Experten getroffen werden sollen.

- Inhaltlich zeigt sich ein signifikanter Effekt: Sowohl unmittelbar nach der Veranstaltung als auch zwei Monate später scheint sich der Standpunkt zum Thema zu verfestigen. Dieser tendiert in Richtung eines positiven Urteils zum Atomausstieg.

- Gruppenunterschiede bei den Aspekten Meinungsbildung und -änderung gibt es kaum. Am deutlichsten wurden diese im Hinblick auf die Meinung gegenüber Wissenschaftlern und Politikern. Hier zeigen sich bei Personen mit niedriger formaler Bildung stärkere Effekte als bei Personen mit höherer formaler Bildung. Das Alter zeigt einen deutlichen Effekt bei der Einschätzung des Bürgereinflusses auf die gesellschaftlichen Entscheidungen. Diesen sehen die älteren Teilnehmer deutlich kritischer als die jüngeren.

\section{Diskussion der Ergebnisse}

Partizipationsverfahren werden häufig unter den Schlagworten Teilhabe, Mitbestimmung und Mitwirkung diskutiert. Ziel ist es in der Regel, eine breite gesellschaftliche Debatte über organisierte Interessen und wissenschaftliche Experten hinaus anzustoßen (Hennen/Petermann/Scherz 2004). Untersuchungen über die Wirkung auf die Teilnehmer können weitere Erkenntnisse über Meinungs- und Akzeptanzfragen ermöglichen. Die Evaluation des Bürgerdialogs zum Thema Energie bestätigt Wirkungseffekte hinsichtlich der Dimensionen Kompetenzerwerb der Befragten und Meinungsbildung und -änderung. Diese Effekte zeigen sich am stärksten bei Personen mit niedriger formaler Bildung.

Kompetenzerwerb: Vor allem das Fakten- und Sachwissen hat nach eigener Einschätzung der Bürger zugenommen. Ein Grund für diesen Effekt ist möglicherweise der Austausch mit den Experten auf den Bürgerkonferenzen. Allerdings kann damit nicht der objektive Wissenszuwachs belegt werden.

Eine ähnliche Einschränkung gilt bei der Frage nach der Zunahme an Interesse und der intrinsischen Motivation, sich weiter mit dem Thema zu beschäftigen. Nicht 
gemessen wurde, ob die verbale Zustimmung wirklich dazu führt, dass sich die Befragten weiter zum Thema Energie informieren, sich mehr engagieren bzw. mit anderen darüber diskutieren und in welcher Form sie dies tun.

Die Aneignung sozialer, demokratischer und wissenschaftlicher Kompetenzen kann mit den vorliegenden Items nur bedingt untersucht werden. Es zeigt sich aber ein Effekt bei der kommunikativen Kompetenz, der nach den Annahmen des kooperativen Lernens sicherlich auf die Kleingruppenarbeit und die Möglichkeit für jeden Bürger, sich einzubringen, zurückzuführen ist.

Einschränkend ist anzumerken, dass bei allen Kompetenz-Items methodologische Effekte denkbar sind. Möglicherweise sind die Operationalisierungen der Items zu allgemein, die Fünfer-Skala zu wenig differenziert und die Antworten sozial erwünscht. Hier stößt die standardisierte Abfrage, die unmittelbar nach der Teilnahme an Partizipationsveranstaltungen für die Bürger zeitlich und kognitiv nicht zu anspruchsvoll sein kann und darf, an ihre Grenzen. Für einen differenzierteren Einblick wäre deshalb die Durchführung von qualitativen Interviews mit einzelnen Bürgern interessant.

Meinungsbildung bzw. -änderung: Auch Effekte auf die Meinungsbildung bzw. -änderung sind messbar. Die Teilnehmer sind nach der Veranstaltung signifikant stärker der Ansicht, dass Bürgerbeteiligung im Entscheidungsprozess die Qualität politischer Entscheidungen verbessert. Der Wert sinkt jedoch zwei Monate nach der Bürgerkonferenz wieder auf sein Ausgangsniveau zurück. Auch die Meinung gegenüber Politikern oder Wissenschaftlern hat sich kaum geändert. Offenbar hat, wie auch andere Evaluationen zeigen, die einmalige Veranstaltung in diesem Bereich keine langfristige Wirkung auf die Meinung der Bürger (Wissenschaft im Dialog 2011: 20). Zurückzuführen ist dies möglicherweise auf die Stabilität politischer Einstellungen (Gabriel/Falter/Rattinger 2005). Allerdings sind die Beteiligten auch ca. zwei Monate nach der Veranstaltung weniger der Ansicht, dass Entscheidungen zu komplexen wissenschaftlich-technischen Themen ausschließlich von Experten getroffen werden sollten. Möglicherweise hängt dies mit dem gewonnenen Faktenund Sachwissen zusammen.

Auch zum Thema Energie zeigen sich signifikante Veränderungen: Im Verlauf der drei Erhebungswellen wird sichtbar, dass sich der Standpunkt der Teilnehmer zum Thema Zukunft der Energie verfestigt (Zimmer 2002; Wissenschaft im Dialog 2011). Im Unterschied zur Meinung über Bürgerbeteiligung scheint dieser Effekt auch eine langfristige Wirkung auf die Teilnehmer zu haben.

Gruppenunterschiede zeigen sich vor allem bei der Dimension Kompetenzerwerb, allerdings nicht bei allen untersuchten Variablen gleichermaßen: 
- Am deutlichsten zeigen sich die Gruppenunterschiede bei der Variable Bildung. Vor allem Personen mit niedriger formaler Bildung geben an, allgemein mehr über das Thema Energie gelernt zu haben und zeigen nach der Veranstaltung mehr Interesse am Thema. Auch die Meinung gegenüber Wissenschaftlern und Politikern hat sich bei dieser Personengruppe am ehesten verändert. Warum genau bei dieser Gruppe die Effekte am stärksten sind, kann hier nur vermutet werden. Eventuell unterliegt diese Gruppe dem Druck sozial erwünschter Antworten stärker als andere Personen. Möglicherweise finden sich auch Zusammenhänge mit spezifischen Persönlichkeitsmerkmalen, die es weiter zu untersuchen gilt. So wird zwar in der jüngeren Partizipationsforschung zunehmend der Einfluss von Persönlichkeitsmerkmalen auf die Bereitschaft zur politischen Partizipation untersucht (Steinbrecher/Schoen 2012), aber die Analyse des Zusammenhangs mit Lerneffekten steht noch aus. Derartige Untersuchungen wären für die Durchführung von politischen Partizipationsveranstaltungen zentral, weil gerade Personen mit niedriger formaler Bildung eher schwer zur Teilnahme an solchen Veranstaltungen zu gewinnen sind (Steinbrecher 2009: 179 ff.). Möglicherweise kann der Lerneffekt erfolgreich für die Umwerbung dieser Zielgruppen eingesetzt werden. Denn „bei vielen potentiell Beteiligten könnte sich die Frage stellen, inwiefern sie sich die Mühe machen und kostbare Zeit opfern sollten, wenn sie persönlich und direkt nicht davon profitieren? Beteiligung wird in solchen Fällen einfach häufig als reine Zeitverschwendung angesehen“ (Wessels 2006: 38).

- Die Variable Alter zeigt wenige Auffälligkeiten. Dies überrascht im Hinblick auf Studien aus dem bürgerschaftlichen Engagement. Denn hier werden Effekte auf sogenannte Soft Skills vor allem bei jüngeren Personen gemessen (Gensicke/ Picot/Geiss 2006). Allerdings wird dort der Zeiteffekt als erklärende Variable hinzugezogen. Denkbar wäre, dass Lernerfolge bei dieser Gruppe verstärkt nach einer gewissen Zeit bzw. einer wiederholten Teilnahme auftreten. Dementsprechend wäre zu vermuten, dass beispielsweise bei Konsensuskonferenzen der altersabhängige Lerneffekt stärker ist.

- Auch die Unterschiede zwischen Männern und Frauen sind selten signifikant. Frauen geben allerdings häufiger an, mehr über das Thema Energie erfahren zu haben. Vor allem einige Wochen nach der Veranstaltung ist dieser Effekt signifikant. Er ist möglicherweise auch themenabhängig, denn bei Energietechnologien haben Männer aufgrund der Nähe zum Thema Technik eventuell mehr Vorkenntnisse (vgl. Pfenning/Schulz 2012). Vielleicht wäre bei anderen Themen der Lerneffekt bei Männern größer als bei Frauen oder der Unterschied wäre zumindest weniger ausgeprägt (z. B. Medizin). 
In diesem Artikel wurde die Frage der Wirkung der Bürgerkonferenzen sehr eng mit Blick auf den Kompetenzerwerb und die Meinungsbildung der Bürger untersucht. Doch Bürgerkonferenzen legitimieren sich nicht nur durch einen Lerneffekt auf Bürgerseite, sondern es geht um Lernprozesse bei allen Beteiligten, also auch bei eingebundenen Experten und politischen Entscheidern. Weitere interessante Fragen wären in diesem Zusammenhang beispielsweise, was die Politiker oder Experten durch die Einbindung und den offenen Austausch mit den Bürgern lernen. Bisherige Studien deuten darauf hin, dass sie unter anderem lernen, die Sichtweisen von Bürgern besser zu verstehen und sich mit ihnen face to face auseinanderzusetzen (Joss 2000).

Über die Dauer bzw. Nachhaltigkeit der Effekte können nur bedingt Aussagen getroffen werden. Inwieweit das Interesse und die Bereitschaft, sich mit dem jeweiligen Thema auseinanderzusetzen, über einen längeren Zeitraum bestehen bleibt, kann nicht beantwortet werden. Zwar deutet die Befragung ca. zwei Monate nach der Bürgerkonferenz auf mittelfristige Effekte beim Bürgerdialog Energietechnologien hin, aber darüber hinaus sind keine Aussagen möglich. Derartige Untersuchungen sind bei Partizipationsverfahren aufgrund der geringen Fallzahl grundsätzlich schwierig (Stollen 2011), denn bei jeder Befragungswelle sinkt der Rücklauf; damit sind aussagekräftige Ergebnisse irgendwann nicht mehr möglich. Unklar bleibt somit auch, inwieweit die Bürger mit der Nutzung ihrer Ergebnisse durch das BMBF zufrieden sind oder Frustration einsetzt, wenn sie das Gefühl haben, die Ergebnisse werden bei politischen Entscheidungen nicht ausreichend berücksichtigt.

Insgesamt bestätigen die Evaluationsergebnisse der Bürgerkonferenzen zum Thema Energie die Ergebnisse anderer Untersuchungen über die Wirkung von politischen Beteiligungsverfahren vergleichbarer Konzeption (Stollen 2011). Auch wenn aus diesen Fallanalysen statistisch keine verallgemeinerbaren Schlüsse möglich sind, deutet sich ein durchgängiges Muster an: Politische Partizipationsformate bieten den Rahmen, Lerneffekte und -anstöße bei den Bürgern anzuregen. Zumindest gilt dies für Veranstaltungen, bei denen die Prinzipien des kooperativen Lernens gelten. Die Einbindung von Experten, die Kleingruppendiskussionen und das Ziel der Entwicklung eines gemeinsamen Statements erscheinen dabei als wichtige Einflussfaktoren. Ob die Wirkung bei anderen Beteiligungsformaten ähnlich ausfällt, vor allem wenn das Ziel nicht die gemeinsame Entwicklung eines Statements ist, muss in weiteren Analysen geprüft werden.

Die Frage, inwieweit die ermittelten Effekte mit dem formalen Partizipationsdesign zusammenhängen, erweist sich auch im Hinblick auf psychologische und medizinische Erkenntnisse als relevant. Vor allem der Zusammenhang mit Emotionen 
ist zukünftig näher zu beleuchten. So zeigen Studien aus der Hirnforschung, dass positive Emotionen wie Hoffnung auf Erfolg den Einsatz von Lernstrategien begünstigen und negative Emotionen wie Langeweile oder Stress das Gegenteil bewirken können (Reinmann-Rothmeier 2003: 6). Daher erscheint es für den Lernerfolg wichtig, den Teilnehmern ein konkretes Mandat der Veranstaltung zu kommunizieren und den Tagesablauf effizient aber machbar zu planen.

Auch beim Thema der Veranstaltung spielen Emotionen eine wichtige Rolle: Fühlen sich die Personen emotional betroffen, dann lernen sie mehr (Roth 2001; Spitzer 2002; Traindl 2007). Dieser Zusammenhang hat zweierlei Konsequenzen: Zum einen kann dies bei der inhaltlichen Gestaltung der Veranstaltung genutzt werden. Persönliche Bezüge und positive Gefühle erhöhen möglicherweise die Bereitschaft, sich aktiv in die Diskussion einzubringen und verbessern gleichzeitig die Lernerfolge. Zum anderen ist das grundsätzliche Thema der Veranstaltung wichtig. Bei anderen Themen wie beispielsweise aus dem Medizin-Bereich ist die emotionale Bedeutung für die Bürger möglicherweise höher und dementsprechend wären die Wirkungseffekte stärker als beim Thema Energietechnologien. Derartige Zusammenhänge zwischen dem Thema der Veranstaltung und der Wirkung sind in vergleichenden Studien weiter zu hinterfragen.

Die Ergebnisse zeigen auch methodische Implikationen für die Konzeption und Durchführung von Evaluationen derartiger Partizipationsverfahren. Vor allem die Kombination qualitativer und quantitativer Verfahren erscheint sinnvoll. Teilnehmende Beobachtungen könnten beispielsweise den Fokus auf die Gruppen legen, bei denen die statistischen Effekte niedriger sind und prüfen, ob es hier einen $\mathrm{Zu}-$ sammenhang mit der Art und Häufigkeit der Beteiligung gibt. So kann geprüft werden, ob alle Bürger fair behandelt wurden, das heißt sich gleichermaßen in die Diskussion einbringen konnten.

\section{Fazit}

Die Ergebnisse der Wirkungsanalyse im Rahmen des Bürgerdialogs „Energietechnologien für die Zukunft" bestätigen, dass es bei Partizipationsverfahren um mehr geht als um politische Mitbestimmung. Die Bürger erlangen neues Wissen, ihr Interesse nimmt zu, sie möchten sich mit dem Thema intensiver auseinandersetzen und sich mehr engagieren.

Allerdings wird das Thema in der bisherigen Forschung noch wenig aufgegriffen. So können mit den bisherigen Studien beispielsweise nur bedingt Aussagen über die Wirkungen unterschiedlicher Beteiligungsformate gezogen werden. Dennoch kann mit den Ergebnissen die Werbetrommel für Partizipationsverfahren gerührt 
und eventuell auch die Teilnahmemotivation erhöht werden. Möglichen Teilnehmern kann damit bereits im Vorfeld signalisiert werden, dass sie auch selbst etwas aus ihrer Teilnahme mitnehmen werden. Allerdings ist es auch wichtig, dass die Ergebnisse bei politischen Entscheidungen berücksichtigt werden, damit negative Wirkungseffekte wie Politikverdrossenheit nicht begünstigt werden.

\section{Literatur}

Abelson, Julia/Forest, Pierre-Gerlier/Eyles, John/Smith, Patricia/Martin, Elizabeth/Gauvin, Francois-Pierre, 2003: Deliberations about deliberative methods. Issues in the design and evaluation of public participation processes, in: Social Science \& Medicine 57, 239-251.

Andersen, Vibeke Normann/Hansen, Kaspar M., 2007: How deliberation makes better citizens. The Danish Deliberative Poll on the Euro, in: European Journal of Political Research 46, 531-556.

Barber, Benjamin, 1984: Strong Democracy, Berkeley.

Beierle, Thomas C./Cayford, Jerry, 2002: Democracy in Practice. Public Participation in Environmental Decisions, Washington, D.C.

Bundesministerium für Bildung und Forschung, 2011: Bürgerdialog Zukunftsthemen, http://www.buergerdialog-bmbf.de/ (Stand: 28.11.2012).

Burow, Olaf-Axel/Kühnemuth, Kathrin, 2004: Brauchen Wissenschaft und Politik Bürgerberatung? Möglichkeiten und Grenzen der Bürgerkonferenz, in: Christof Tannert/Peter Wiedemann (Hrsg.), Stammzellen im Diskurs. Ein Lese- und Arbeitsbuch zu einer Bürgerkonferenz, München, 117-129.

Chambers, Simone, 1996: Reasonable Democracy, Ithaca.

Curran, Patrick J./West, Stephan G./Finch, John, 1996: The robustness of test statistics to nonnormality and specification error in confirmatory factor analysis, in: Psychological Methods 1, 16-29.

Delli Carpini, Michael X./Cook, Fay Lomax/Jacobs, Lawrence R., 2004: Public Deliberation, Discursive Participation, and Citizen Engagement. A Review of the Empirical Literature, in: Annual Review of Political Science 7 (1), 315-344. Derichs-Kunstmann, Karin, 2001: Lernen Frauen anders? Empirische Befunde zur Inszenierung des Geschlechterverhältnisses in Lernsituationen, in: Gender-Qualifizierung für die Bildungsarbeit, Arbeitsmaterial Nr. 1, http://www.genderqualifizierung.de/links/Derichs-Kunstmann\%20Materialien\%201.pdf (Stand: 13.6.2013).

Gabriel, Oscar W./Holtmann, Everhard, 2005: Das politische System der Bundesrepublik Deutschland, München/Wien. 
Gabriel, Oscar W./Falter, Jürgen W./Rattinger, Hans, 2005: Wächst zusammen, was zusammen gehört? Stabilität und Wandel politischer Einstellungen im wiedervereinigten Deutschland, Baden-Baden.

Geißel, Brigitte, 2008: Wozu Demokratisierung der Demokratie? Kriterien zur Bewertung partizipativer Arrangements, in: Angelika Vetter (Hrsg.), Erfolgsbedingungen lokaler Bürgerbeteiligung, Wiesbaden, 29-48.

Gensicke, Thomas/Picot, Sibylle/Geiss, Sabine, 2006: Freiwilliges Engagement in Deutschland 1999-2004. Ergebnisse der repräsentativen Trenderhebung zu Ehrenamt, Freiwilligenarbeit und bürgerschaftlichem Engagement, Wiesbaden.

Gohl, Christopher/Wüst, Jürgen, 2008: Beteiligung braucht Wissen - Beteiligung schafft Wissen, in: Angelika Vetter (Hrsg.), Erfolgsbedingungen lokaler Bürgerbeteiligung, Wiesbaden, 259-280.

Goldschmidt, Rüdiger/Scheel, Oliver/Renn, Ortwin, 2012: Zur Wirkung und Effektivität von Dialog- und Beteiligungsformaten, in: Stuttgarter Beiträge zur Risikound Nachhaltigkeitsforschung 23.

Green, Norm/Green, Kathy, 2006: Kooperatives Lernen im Klassenraum und im Kollegium. Das Trainingsbuch, Großburgwedel.

Gutmann, Amy/Thompson, Dennis, 1996: Democracy and Disagreement, Cambridge.

Habermas, Jürgen, 1992: Drei normative Modelle der Demokratie. Zum Begriff deliberativer Demokratie, in: Herfried Münkler (Hrsg.), Die Chancen der Freiheit. Grundprobleme der Demokratie, München und Zürich, 11-24.

Hebestreit, Ray, 2013: Partizipation in der Wissensgesellschaft. Funktion und Bedeutung diskursiver Beteiligungsverfahren, Wiesbaden.

Hennen, Leonhard/Petermann, Thomas/Scherz, Constanze, 2004: Partizipative Verfahren der Technikfolgenabschätzung und parlamentarische Politikberatung. Neue Formen der Kommunikation zwischen Wissenschaft, Politik und Öffentlichkeit, in: TAB-Arbeitsbericht 96, http://www.tab-beim-bundestag.de/ de/pdf/publikationen/berichte/TAB-Arbeitsbericht-ab096.pdf

(Stand: 29.1.2013).

Holtkamp, Lars/Bogumil, Jörg/Kißler, Leo, 2006: Kooperative Demokratie. Das politische Potenzial von Bürgerengagements, Frankfurt a. M./New York.

Joss, Simon, 2000: Participation in parliamentary technology assessment, in: Norman J. Vig/Herbert Paschen (Hrsg.), Parliaments and Technology. The development of technology assessment in Europe, Albany, 325-362.

Kersting, Norbert, 2008: Evaluation dialogischer Beteiligungsinstrumente, in: Norbert Kersting (Hrsg.), Politische Beteiligung. Einführung in dialogorientierte 
Instrumente politischer und gesellschaftlicher Partizipation, Wiesbaden, 270-292.

Kubicek, Herbert/Lippa, Barbara/Koop, Alexander, 2011: Erfolgreich beteiligt?

Nutzen und Erfolgsfaktoren internetgestützter Bürgerbeteiligung. Eine empirische Analyse von 12 Fallbeispielen, Gütersloh.

Lösch, Bettina, 2005: Deliberative Politik. Moderne Konzeptionen von Öffentlichkeit, Demokratie und politischer Partizipation, Münster.

Luskin, Robert C./Fishkin, James S./Iyengar, Shanto, 2004: Considered opinions on U.S. foreign policy. Face-to-face versus online deliberative polling. Paper presented at the International Communication Association Conference, New Orleans, http://spp.nus.edu.sg/ips/docs/events/Deliberative_Polling_background \%20materials.pdf (Stand: 29.1.2013).

Luskin, Robert C./Fishkin, James S./Jowell, Roger, 2002: Considered Opinions. Deliberative Polling in Britain, in: British Journal of Political Science 32, 455-487.

Marxer, Wilfried, 2004: „Wir sind das Volk“: Direkte Demokratie. Verfahren, Verbreitung, Wirkung. Schriftliche Fassung des Vortrages am Liechtenstein-Institut vom 2. November 2004 in der Vorlesungsreihe „Herausforderung Demokratie“, http://www.liechtenstein-institut.li/Portals/0/contortionistUniverses/408/rsc/Pu blikation_downloadLink/LIB_024.pdf (Stand: 15.1.2013).

Mayer, Igor/de Vries, Jolanda/Geurts, Jac, 1995: An evaluation of the effects of participation in an consensus conference, in: Simon Joss/John Durant (Hrsg.), Public participation in science. The role of consensus conferences in Europe, London, 109-124.

Mendelberg, Tali, 2002: The Deliberative Citizen: Theory and Evidence, in: Michael X. Delli Carpini/Leonie Huddy/Robert Y. Shapiro (Hrsg.), Political Decisionmaking, Deliberation and Participation, in: Research in Micropolitics 6, Greenwich, 151-193.

Min, Seong-Jae, 2007: Online vs. face-to-face deliberation. Effects on civic engagement, in: Journal of Computer-Mediated Communication 12 (4), 1369-1387.

Newig, Jens, 2011: Partizipation und Kooperation zur Effektivitätssteigerung in Politik und Governance?, in: Harald Heinrichs/Katina Kuhn/Jens Newig (Hrsg.), Nachhaltige Gesellschaft. Welche Rolle für Partizipation und Kooperation?, Wiesbaden, 65-79.

Niehoff, Erika, 1993: Lernbedingungen von Frauen im Bereich gewerblich-technischer Umschulungen, in: Wiltrud Giesecke (Hrsg.), Feministische Bildung. Frauenbildung, Pfaffenweiler, 79-89. 
Pfenning, Uwe/Schulz, Marlen, 2012: Gender(a)symmetrie im MINT-Bereich, in: Uwe Pfenning/Ortwin Renn (Hrsg.), Wissenschafts- und Technikbildung auf dem Prüfstand. Zum Fachkräftemangel und zur Attraktivität der MINT-Bildung und -Berufe im europäischen Vergleich, Baden-Baden, 143-157.

Reed, Mark S./Evely, Anna C./Cundill, Georgina/Fazey, Ioan R. A./Glass, Jayne/ Laing, Adele/Newig, Jens/Parrish, Brad/Prell, Christina/Raymond, Chris/Stringer, Lindsay C., 2010: What is Social Learning?, in: Ecology and Society 15 (4), r1.

Reinmann-Rothmeier, Gabi, 2003: Die vergessenen Weggefährten des Lernens. Herleitung eines Forschungsprogramms zu Emotionen beim E-Learning, in: Medienpädagogik Arbeitsberichte, Arbeitsbericht Nr. 1, http://opus.bibliothek. uni-augsburg.de/opus4/frontdoor/index/index/docId/369 (Stand: 13.6.2013).

Roth, Gerhard, 2001: Fühlen, Denken, Handeln. Wie das Gehirn unser Verhalten steuert, Frankfurt a. M.

Rowe, Gene/Frewer, Lynn J., 2000: Public Participation Methods. A Framework for Evaluation, in: Science, Technology, \& Human Values 25, 3-29.

Rowe, Gene/Marsh, Roy/Frewer, Lynn J., 2004: Evaluation of a deliberative conference, in: Science, Technology, \& Human Values 29, 88-121.

Schmidt, Claudia, 1988: Typisch weiblich - typisch männlich. Geschlechtstypisches Kommunikationsverhalten in studentischen Kleingruppen, Tübingen.

Schophaus, Malte/Dienel, Liudger, 2002: Bürgerausstellung. Ein neues Beteiligungsverfahren für die Stadtplanung, in: Forschungsjournal Neue Soziale Bewegungen 15, 90-96.

Selle, Klaus, 1996: Planung und Kommunikation. Gestaltung von Planungsprozessen in Quartier, Stadt und Landschaft. Grundlagen, Methoden, Praxiserfahrungen, Wiesbaden.

Selle, Klaus, 2000: Was? Wer? Wie? Warum? Voraussetzungen und Möglichkeiten einer nachhaltigen Kommunikation, Dortmund.

Sellke, Piet/Renn, Ortwin/Cornelisse, Corinne, 2007: European Citizens' Panel. Final Report of the External Evaluation, in: Stuttgarter Beiträge zur Risiko- und Nachhaltigkeitsforschung 7, 1-98.

Spitzer, Manfred, 2002: Gehirnforschung und die Schule des Lebens, Heidelberg. Steinbrecher, Markus, 2009: Politische Partizipation in Deutschland, Baden-Baden. Steinbrecher, Markus/Schoen, Harald, 2012: Persönlichkeit und politische Partizipation im Umfeld der Bundestagswahl 2009, in: Politische Psychologie 2, 58-74. Stollen, Torsten, 2011: Deliberation als Brücke zwischen passiver und aktiver Öffentlichkeit. Ein Feldexperiment zu den Chancen und Grenzen verschiedener Formen der Bürgerbeteiligung in der deutschen Gesundheitspolitik, Berlin. 
Stoltenberg, Ute, 2007: Gesellschaftliches Lernen und Partizipation, in: Helga Jonuschat/Elke Baranek/Maria Behrendt/Kristina Dietz/Bianca Schlußmeier/Heike Walk/Andreas Zehm (Hrsg.), Partizipation und Nachhaltigkeit. Vom Leitbild zur Umsetzung, München, 54-66.

Talpin, Julien/Wojcik, Stéphanie, 2010: Deliberating Environmental Policy Issues. Comparing the Learning Potential of Online and Face-To-Face Discussions on Climate Change, in: Policy \& Internet 2 (2), 61-93.

Traindl, Arndt, 2007: Neuromarketing. Die innovative Visualisierung von Emotionen, Linz.

Urban, Dieter/Mayerl, Jochen, 2008: Regressionsanalyse. Theorie, Technik und Anwendung, Wiesbaden.

Werner, Fridjof, 1983: Gesprächsverhalten von Frauen und Männern, Frankfurt a. M.

Wessels, Heike, 2006: Unter welchen Bedingungen ist Öffentlichkeitsbeteiligung effektiv? Gesellschaftlicher Kontext, soziale Normen und politische Kultur, in: Jens Newig/Oliver Fritsch (Hrsg.), Effektivität von Beteiligungsprozessen, Osnabrück, 35-45.

Wissenschaft im Dialog gGmbH, 2011: Abschlussbericht Forschungsprojekt „Wissenschaft debattieren!“, Berlin, http://www.wissenschaft-im-dialog.de/fileadmi n/redakteure/dokumente/Wissenschaft_debattieren/Abschlussbericht-Finalweb .pdf (Stand: 15.6.2012).

Zimmer, Réne, 2002: Begleitende Evaluation der Bürgerkonferenz „Streitfall Gendiagnostik“, Karlsruhe, http://isi.fraunhofer.de/isi-de/publ/download/isi02b11/ gendiagnostik.pdf (Stand: 9.7.2013).

Korrespondenzanschrift:

Dr. Marlen Niederberger

Verena Keierleber, B.A.

Isabel Schneider, M.A.

Zentrum für Interdisziplinäre Risiko- und Innovationsforschung der Universität Stuttgart (ZIRIUS)

Seidenstraße 36

70174 Stuttgart

E-Mail: marlen.niederberger@sowi.uni-stuttgart.de;

verena.keierleber@sowi.uni-stuttgart.de; isabel.schneider@sowi.uni-stuttgart.de 